\title{
Time-resolved smoothness of distributed brain activity tracks conscious states and unifies emergent neural phenomena
}

\section{Aditya Nanda}

Vanderbilt University

Graham Johnson

Vanderbilt University

\section{Yu Mu}

Chinese Academy of Science

\section{Misha Ahrens}

Janelia Research Campus, Howard Hughes Medical Institute https://orcid.org/0000-0002-3457-4462

\section{Catie Chang}

Vanderbilt University https://orcid.org/0000-0003-1541-9579

\section{Dario Englot}

Vanderbilt University Medical Center

\section{Michael Breakspear}

University of Newcastle, NSW https://orcid.org/0000-0003-4943-3969

Mikail Rubinov ( $\nabla$ mika.rubinov@vanderbilt.edu )

Vanderbilt University

\section{Article}

Keywords:

Posted Date: December 29th, 2021

DOI: https://doi.org/10.21203/rs.3.rs-1195514/v1

License: (1) (1) This work is licensed under a Creative Commons Attribution 4.0 International License.

Read Full License 


\section{Time-resolved smoothness of distributed brain activity tracks conscious states and unifies emergent neural phenomena}

Aditya Nanda ${ }^{1}$,

Graham W Johnson ${ }^{1}$,

$\mathrm{Yu} \mathrm{Mu}$,

Misha B Ahrens ${ }^{5}$,

Catie Chang ${ }^{1,3,4}$,

Dario J Englot ${ }^{1,6,7}$,

Michael Breakspear ${ }^{8,9}$,

Mikail Rubinov ${ }^{1,4,3, *}$

${ }^{1}$ Department of Biomedical Engineering, Vanderbilt University, USA

${ }^{2}$ Institute of Neuroscience, Chinese Academy of Sciences, China

${ }^{3}$ Department of Electrical and Computer Engineering, Vanderbilt University, USA

${ }^{4}$ Department of Computer Science, Vanderbilt University, USA

${ }^{5}$ Janelia Research Campus, Howard Hughes Medical Institute, USA

${ }^{6}$ Department of Neurological Surgery, Vanderbilt University Medical Center, USA

${ }^{7}$ Department of Radiology and Radiological Sciences, Vanderbilt University Medical Center, USA

${ }^{8}$ School of Psychology, University of Newcastle, Australia

${ }^{9}$ School of Medicine and Public Health, University of Newcastle, Australia

${ }^{*}$ Corresponding author (mika.rubinov@ vanderbilt.edu) 


\section{Abstract}

Much of systems neuroscience posits that emergent neural phenomena underpin important aspects of brain function. Studies in the field variously emphasize the importance of distinct emergent phenomena, including weakly stable dynamics, arrhythmic $1 / f$ activity, long-range 5 temporal correlations, and scale-free avalanche statistics. Few studies, however, have sought to reconcile these often abstract phenomena with interpretable properties of neural activity. Here, we developed a method to efficiently and unbiasedly generate model data constrained by interpretable empirical features in long neurophysiological recordings. We used this method to ground several major emergent neural phenomena to time-resolved smoothness, the correlation of distributed brain activity between adjacent timepoints. We first found that in electrocorticography recordings, time-resolved smoothness closely tracked transitions between conscious and anesthetized states. We then showed that a minimal model constrained by timeresolved smoothness, variance, and mean, captured dynamical and statistical emergent neural phenomena across modalities and species. Our results thus decouple major emergent neural phenomena from network mechanisms of brain function, and instead couple these phenomena to spatially nonspecific, time-resolved changes of brain activity. These results anchor several theoretical frameworks to a single interpretable property of the neurophysiological signal and, in this way, ultimately help bridge abstract theories of brain function with observed properties of brain activity. 


\section{Introduction}

Coordinated neuronal interactions give rise to intricate patterns of distributed brain activity. Much of systems neuroscience seeks to understand the spatiotemporal structure of these patterns. Studies often seek to explain this structure in terms of emergent neural phenomena, spatiotemporal patterns of brain activity that reflect complex interactions of individual neurons or brain regions. Many of these phenomena are situated within longstanding theoretical frameworks $[1-4]$.

One such framework centers on the study of dynamical phenomena $[3,5,6]$. Dynamical phenomena show up as trajectories in phase-space embeddings of neuronal $[7,8]$ or regional [9] recordings. These embeddings provide a convenient geometric representation of distributed brain activity [10]. Moreover, the smoothness of dynamical trajectories can endow dynamical activity with robustness to high-dimensional noise [11].

Another framework centers on the study of scale-free statistical phenomena. Scale-free phenomena lack typical sizes, durations, or frequencies $[2,12,13]$ and show up as heavytailed functions or distributions that resemble straight lines on log-log plots [14]. One example is the arrhythmic $1 / f$ activity of local field potentials $[15,16]$. Another is the heavy-tailed distributions of sizes and durations of synchronous bursts or avalanches of neurons [17-19] and brain regions [20, 21]. Scale-free phenomena associate with long-range temporal correlations of neural activity [22] and imply theoretically appealing function, including efficient representation of sensory input $[12,23]$.

A third framework, the theory of neural criticality, lies at the intersection of the first two, and seeks to explain both dynamical and statistical emergent phenomena. This framework proposes that neural dynamics critically balance on the border of stable and unstable states $[24,25]$. Critical dynamics show highly varying responses to perturbations [26] and give rise to scale-free power spectra, scale-free avalanche statistics and long-range temporal correlations $[27,28]$. Criticality has many theoretically appealing properties, including optimized detection $[29,30]$ and transformation $[17,31]$ of sensory input.

Collectively, these theoretical frameworks underpin a translational literature that seeks to explain the nature of brain function $[2,3]$ and dysfunction $[32,33]$. Studies within this litera- 
ture tend to associate healthy brain activity with optimally structured emergent phenomena, including low-dimensional dynamics [34], scale-free statistics [35], and neural criticality [36]. Similarly, they tend to associate deviations from this optimality with clinically relevant changes in brain activity, including disrupted neuronal excitability [37], loss of consciousness [38], and seizures [39].

However, despite this large and growing body of existing literature, the function and importance of many emergent neural phenomena remains contested or controversial [28, 40, 41]. For example, there is a lack of experimental evidence that reconciles weakly stable dynamics and scale-free statistics with stable and scale-specific circuit mechanisms of cognition or behavior $[42,43]$. In contrast, most support for these and other emergent phenomena comes in three main forms. First, many studies confirm these phenomena against weak null models, typically generated with naive shuffling of empirical data. Such tests do not generally constitute strong evidence of function or importance $[14,40]$. Second, many studies report correlations between emergent phenomena and aspects of brain function $[15,17,38]$. These studies are interesting but often do not fully account for confounding explanations. Third, many studies explain the origin or nature of emergent phenomena in computational models [44-47]. These studies, while also interesting, often require additional generative assumptions that can be difficult to validate. Collectively, the combined presence of strong claims about the nature or importance of emergent neural phenomena, and the absence of similarly strong supporting evidence for these claims, has resulted in an extensive literature of intriguing but inconclusive findings.

Here, we make major steps to resolve this impasse by advancing the approach of surrogate data. This approach evaluates phenomena of interest in model datasets constrained by predefined empirical features, and is increasingly adopted to evaluate features of brain network or population activity $[48,49]$. The approach has several important strengths. First, in contrast to tests against weak null models, it admits strong competing explanations. Second, in contrast to correlational studies, it closely controls for confounds by a priori constraining only a predefined set of features. Finally, in contrast to computational modeling studies, it makes no mechanistic assumptions, beyond assuming the importance of constrained features. 
In contrast to these strengths, computational cost is a major weakness of this approach. More specifically, the generation of highly constrained model data in large recordings can often be prohibitively slow. Here, we overcame this problem by developing a fast and unbiased method for generating model data with a range of interpretable empirical features. In long neurophysiological recordings of distributed brain activity, our method outperforms standard constrained randomization (shuffling) methods by orders of magnitude. This method allowed us to rigorously evaluate emergent neural phenomena in recordings with hundreds of thousands, and in some cases millions, of timepoints across species and modalities.

We used this method to ground several major dynamical and statistical neural phenomena to time-resolved smoothness, the correlation between patterns of distributed activity at adjacent timepoints. First, we found that in electrocorticography recordings, time-resolved smoothness reliably tracked brain-state changes across consciousness and anesthesia. Second, we went beyond correlations to show that a minimal model informed by time-resolved smoothness, variance, and mean, captured major dynamical and statistical emergent neural phenomena across species and modalities. Finally, we showed that five alternative models of the neurophysiological signal, including models with many more constraints, could not explain emergent phenomena to the same extent. Collectively, our results anchor several disparately studied emergent neural phenomena to a single and interpretable property of the neurophysiological signal. We conclude with a discussion of ways by which these results bridge theoretical frameworks with empirical observations.

\section{Results}

\section{Summary of datasets}

Table 1 summarizes the properties of all our studied datasets. We focused our analysis and modeling on recordings with intracranial EEG modalities, namely stereotactic EEG and electroencephalography. Intracranial EEG recordings are optimally suited for the study of emergent phenomena by their combination of millimeter-scale spatial resolution, millisecond-scale temporal resolution, and distributed cortical coverage [54]. In practice, we analyzed and mod- 
Table 1: Summary of analyzed and modeled datasets

\begin{tabular}{|c|c|c|c|c|}
\hline Species, experiment & Modality & Nodes & $\begin{array}{l}\text { Tested emergent phenomena, } \\
\text { sampling rate }\end{array}$ & $\begin{array}{l}\text { Datasets } \\
\text { (nodes } \times \text { timepoints) }\end{array}$ \\
\hline $\begin{array}{l}\text { Macaque monkey, } \\
\text { anesthesia [50] }\end{array}$ & Electrocorticography & \multirow[t]{2}{*}{$\begin{array}{l}\text { Grid electrodes } \\
\text { covering a cortical } \\
\text { hemisphere. }\end{array}$} & $\begin{array}{l}\text { Dynamical phenomena, } 1000 \mathrm{~Hz} \text {. } \\
\text { NB: We followed previous work } \\
\text { that established this sampling rate } \\
\text { as a lower bound for detection } \\
\text { of weakly stable dynamics in } \\
\text { empirical data [38]. }\end{array}$ & $\begin{array}{l}128 \times(3.59 \text { million }) \\
128 \times(3.68 \text { million }) \\
128 \times(3.69 \text { million }) \\
128 \times(3.71 \text { million })\end{array}$ \\
\hline $\begin{array}{l}\text { Macaque monkey, } \\
\text { resting state [50] }\end{array}$ & Electrocorticography & & \multirow{3}{*}{$\begin{array}{l}\text { Statistical phenomena, } 250 \mathrm{~Hz} \text {. } \\
\text { NB: All our results were robust } \\
\text { to analysis and modeling of data } \\
\text { at } 1000 \mathrm{~Hz} \text {, but this higher } \\
\text { sampling rate incurred } \\
\text { unnecessary computational cost. }\end{array}$} & $\begin{array}{l}128 \times(225 \text { thousand }) \\
128 \times(225 \text { thousand }) \\
128 \times(225 \text { thousand }) \\
128 \times(225 \text { thousand })\end{array}$ \\
\hline $\begin{array}{l}\text { Humans, working } \\
\text { memory task [51] }\end{array}$ & Electrocorticography & $\begin{array}{l}\text { Grid electrodes in } \\
\text { parts of frontal and } \\
\text { temporal cortex. }\end{array}$ & & $\begin{array}{l}84 \times(225 \text { thousand }) \\
110 \times(191 \text { thousand }) \\
106 \times(210 \text { thousand }) \\
126 \times(204 \text { thousand })\end{array}$ \\
\hline $\begin{array}{l}\text { Humans, } \\
\text { resting-state [52] }\end{array}$ & Stereotactic EEG & $\begin{array}{l}\text { Implanted electrodes } \\
\text { in cortical regions. }\end{array}$ & & $\begin{array}{l}148 \times(225 \text { thousand }) \\
157 \times(225 \text { thousand }) \\
166 \times(225 \text { thousand }) \\
186 \times(225 \text { thousand })\end{array}$ \\
\hline $\begin{array}{l}\text { Zebrafish, fictive } \\
\text { swimming [53] }\end{array}$ & $\begin{array}{l}\text { Light-sheet calcium } \\
\text { imaging }\end{array}$ & $\begin{array}{l}\text { Neurons distributed } \\
\text { over the whole brain. }\end{array}$ & $\begin{array}{l}\text { Some statistical phenomena, } 3 \mathrm{~Hz} \text {. } \\
\text { NB: We followed previous work } \\
\text { to adopt a modified avalanche } \\
\text { detection procedure most suitable } \\
\text { for analysis of brain-wide light- } \\
\text { sheet calcium imaging data [37]. }\end{array}$ & $\begin{array}{l}(110 \text { thousand }) \times 7100 \\
(117 \text { thousand }) \times 5200\end{array}$ \\
\hline
\end{tabular}


eled datasets that comprised hundreds of thousands, and in some cases millions, of timepoints recorded from around 100 implanted (stereotactic EEG) or grid (electrocorticography) cortical electrodes. In addition to these recordings, we also followed the recent literature to evaluate critical emergent phenomena in light-sheet calcium imaging of fictively swimming zebrafish [37]. The spatiotemporal resolution of light-sheet calcium imaging data provides a unique opportunity to model brain-wide activity with cellular resolution [55]. Nonetheless, the large number of neurons, the small number of timepoints, and the low temporal resolution of these data presented unique challenges in modeling these data, as we discuss below.

\section{Time-resolved smoothness tracks conscious states}

We defined time-resolved smoothness as $\operatorname{corr}\left(\mathbf{v}_{i}, \mathbf{v}_{i+l a g}\right)$, where corr denotes the Pearson correlation coefficient, $\mathbf{v}_{i}$ denotes a vector of neuronal or regional activity at time $i$, and lag denotes an integer number of timepoints. Figure 1a-d shows the distribution of this quantity for immediately adjacent timepoints (lag $=1$ ), grouped separately for each species and modality. The figure shows that time-resolved smoothness was unimodally distributed close to its maximal value of 1 , reflecting gradual changes in distributed brain activity over time. The medians [95\% uncertainty intervals] of this quantity were similar for all intracranial EEG modalities. They were 0.985 [0.953, 0.998] for human stereotactic EEG; 0.985 [0.936, 0.996] for human electrocorticography; 0.964 [0.875, 0.994] for macaque electrocorticography resting-state (sampled at $250 \mathrm{~Hz}$ ), and 0.988 [0.957, 0.996] for macaque anesthesia (sampled at $1000 \mathrm{~Hz}$ ). The values were somewhat lower, 0.930 [0.845, 0.958], for zebrafish calcium imaging (sampled at 3Hz). Extended Data Figures 1-4 show the relationship of time-resolved smoothness and basic properties of the neurophysiological signal.

We adopted the approach of surrogate data to evaluate six empirically constrained models of dynamical and statistical emergent phenomena. The principles of this approach are simple. First, we defined, separately for each model, a set of empirical features, such as timeresolved smoothness or the regional power spectrum. Second, we generated ensembles of model datasets that matched the values of these features, but that were otherwise maximally 


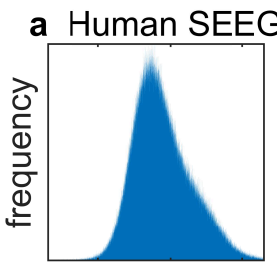

0.90 .990 .999 smoothness

e constraints: time-resolved smoothness, var. and mean. method: nullspace sampling.
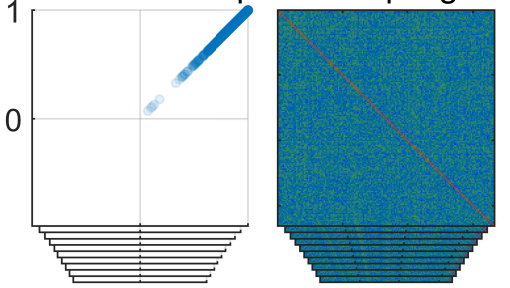

f constraints: time-resolved var.
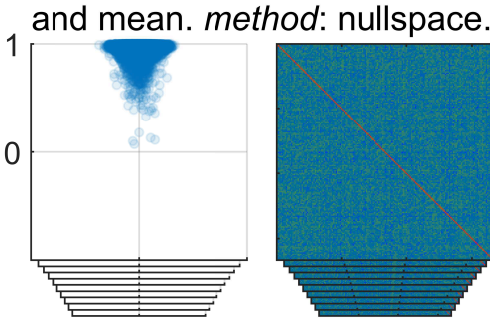

g constraints: time-resolved mean.

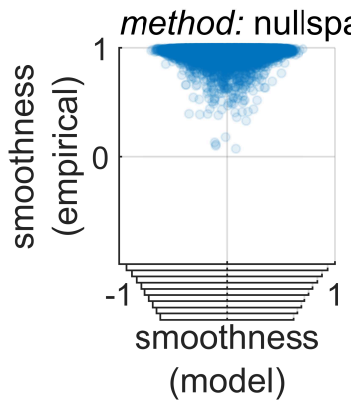

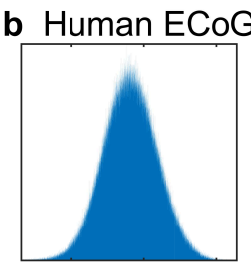

$0.9 \quad 0.990 .999$

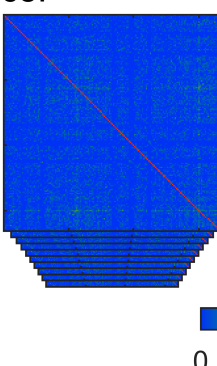

0 c Macaque ECoG

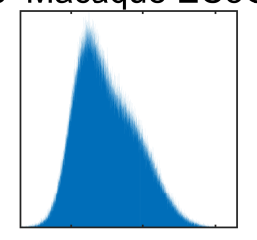

0.90 .990 .999 d Zebrafish $\mathrm{Ca}$

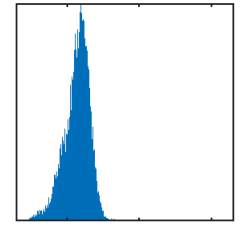

0.90 .990 .999

h constraints: power spectrum/ all-pairwise cross correlation. method: phase randomization.

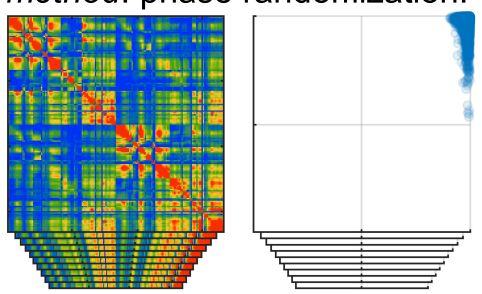

i constraints: timepoint activity. method: frame randomization.

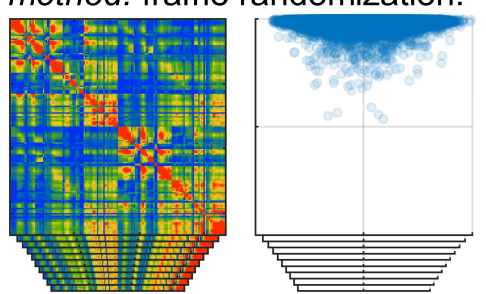

j constraints: cluster variance and mean. method: nullspace.
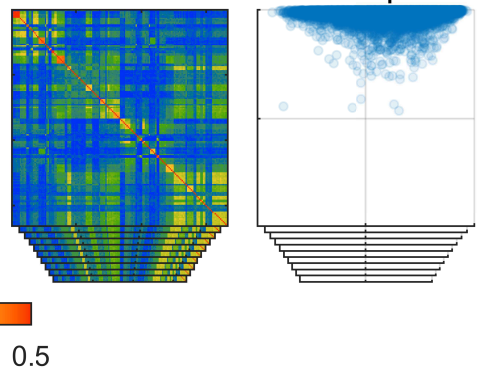

Figure 1: (a-d). Histograms of time-resolved smoothness pooled across all recordings, separately for each species and modality. Note the logarithmic x-axes. Extended Data Figures 1-4 show the relationship of time-resolved smoothness to other basic properties of the neurophysiological signal for individual modalities. (e-j) Properties of (e) smoothness, (f) varmean, (g) mean, (h) power spectrum, (i) shuffled frames, and (j) varmean clusters model data of a single representative recording (human stereotactic EEG). Scatter plots show the correspondence between empirical and model time-resolved smoothness. By definition, this correspondence is exact for smoothness model data. Matrices show all-pairwise node correlations. By definition, these matrices match empirical correlations exactly for powerspectrum and shuffled-frames model data. Abbreviations: SEEG, stereotactic EEG; ECoG, electrocorticography; $\mathrm{Ca}$, calcium imaging. 
random. Third, we evaluated the presence of dynamical and statistical emergent phenomena in empirical and model datasets.

The approach of surrogate data can be prohibitively slow for nontrivial constraints in large datasets. Here, we used insights from linear algebra to develop an unbiased and efficient nullspace sampling method for generating model timeseries with a range of interpretable constraints. The Methods section describes our method in considerable detail. We adopted this method, alongside more standard phase and frame randomization methods $[17,56]$, to define six competing models of the neurophysiological signal. For convenience, we refer to each model using an italicized shorthand. Below, we summarize the properties of each model. We also list the number of model constraints in terms of the number of nodes $n$, the number of timepoints $t$, and the percentage of total data elements.

\section{Model data generated using the nullspace sampling method}

1. smoothness: $3 t(\sim \% 3)$ constraints. Model data constrained by lag $=1$ smoothness, variance, and mean of distributed activity at each timepoint. This was the only model that recapitulated all our considered dynamical and statistical neural emergent phenomena across datasets. A modified version of this model with $12 t(\sim \% 0.01)$ constraints was used to model zebrafish calcium imaging data, as discussed below.

2. varmean: $2 t(\sim \% 2)$ constraints. Model data constrained by the variance and mean of distributed activity at each timepoint. This model allowed us to isolate the contribution of time-resolved smoothness relative to more basic properties of the neurophysiological signal.

3. mean: $t(\sim \% 1)$ constraints. Model data constrained by the mean of distributed activity at each timepoint. This model represents a commonly used weak null model of the neurophysiological signal.

4. varmean clusters: approximately $t(\sim \% 1)$ constraints. Model data constrained by the variance and mean of distributed activity within spatiotemporal clusters. This model denotes a basic low-dimensional representation of the data. A modified version of this 
model with $8 t(\sim \% 0.008)$ constraints was used to model zebrafish calcium imaging data, as discussed below.

\section{Model data generated using phase or frame randomization methods}

5. power spectrum: more than $\frac{1}{2} n t(\% 50)$ constraints. Model data constrained by the power spectrum of each node, and by all-pairwise node cross-correlations. This highly constrained model is often used to evaluate the presence of dynamic correlations in the neuroimaging literature [57]. We found that, in spite of its many constraints, this model did not recapitulate all evaluated emergent phenomena across datasets.

6. shuffled frames: more than $n t(\% 100)$ constraints. Model data constrained by shuffling of individual time frames. This model preserves the exact distributed activity of each timepoint, as well as all-pairwise node correlations.

Figure 1e-j illustrates the extent with which these models captured empirical time-resolved smoothness, as well as all-pairwise node correlations, in a representative recording. This figure allows us to make two important observations. First, it shows that smoothness model data (Figure 1e) does not capture node correlations, in contrast to power-spectrum and shuffledframes model data (Figure 1h-i). Pairwise node correlations reflect, indirectly, mechanistic network interactions that underpin cognition and behavior [58]. Second, it shows that no model data, apart from smoothness model data, captured empirical time-resolved smoothness. These observations imply that time-resolved smoothness reflects spatially nonspecific changes of brain activity, and that these changes are distinct from spatially specific network mechanisms.

To further interpret these changes, we examined the dynamics of time-resolved smoothness in four electrocorticography recordings of macaque monkeys across consciousness and propofol anesthesia [59]. Previous work has shown that emergent properties of brain activity, including slope of the $1 / f$ power spectrum [60-62], as well as dynamical stability [38, 63], separated consciousness and anesthesia in these recordings. Figure $2 a-d$ shows that timeresolved smoothness, a simpler and decidedly non scale-free property (Figure 1a-d) likewise robustly tracked changes in consciousness across all four recordings. The median [95\% uncer- 

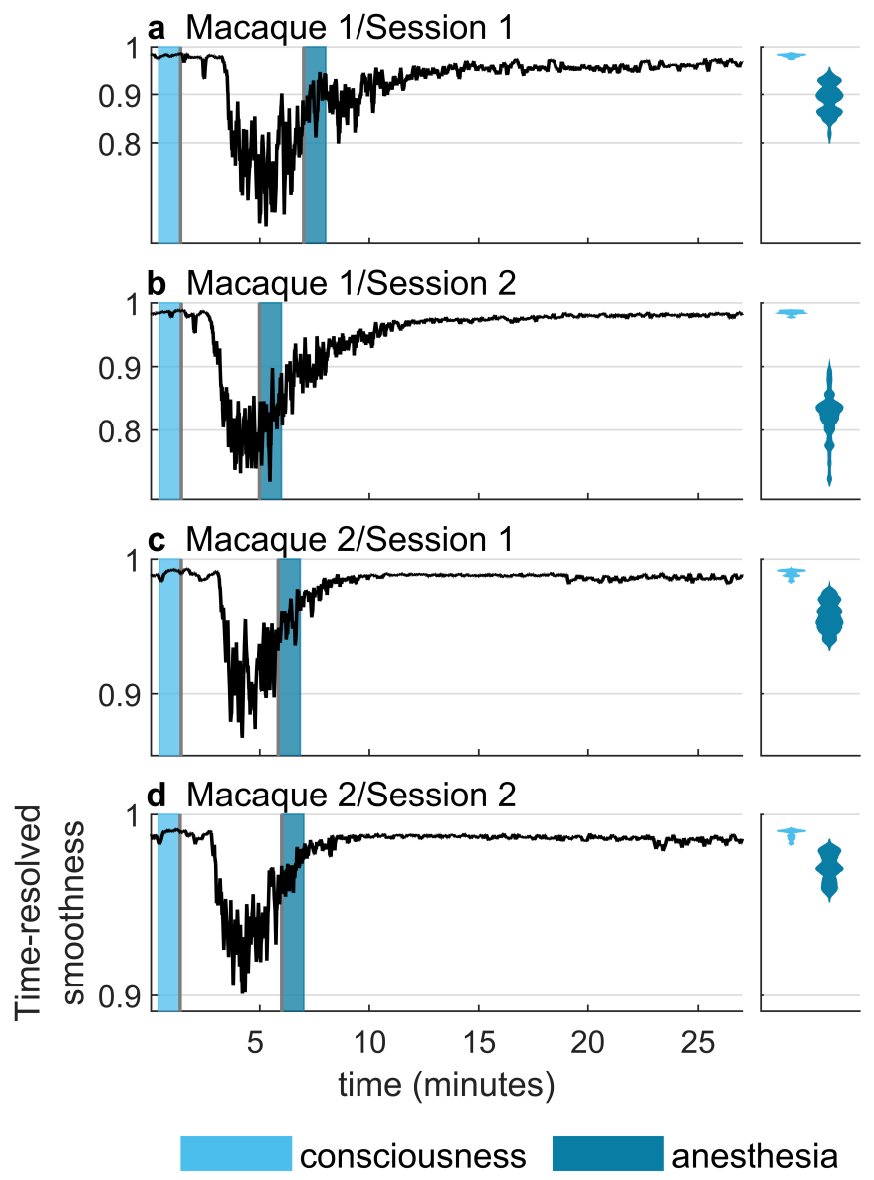

Figure 2: Changes in time-resolved smoothness track changes between consciousness and propofol anesthesia across two electrocorticography recordings of two monkeys (a-d). (Left) Light blue intervals denote a one-minute interval immediately prior to injection of propofol. Dark blue intervals denote one-minute periods immediately following onset of anesthesia based on video observation. (Right) Violin plots of time-resolved smoothness densities within shaded light-blue and dark-blue periods. 
tainty interval] time-resolved smoothness pooled over the four recordings was 0.987 [0.978, $0.992]$ in the one-minute interval preceding injection of propofol, and $0.939[0.788,0.976]$ in the one-minute interval immediately following the onset of anesthesia based on video observation [38] ( $p<10^{-10}$, Wilcoxon rank sum test). Indeed, this metric dropped to even lower values at the peak effect time of propofol, roughly two minutes after injection [64]. Finally, the smaller relative drop of time-resolved smoothness in macaque 2 (Figure $2 \mathrm{c}-\mathrm{d}$ ) matched previous observations that this monkey was not fully anesthetized [63]. Collectively, these results show that time-resolved smoothness can robustly track gross changes in brain activity across conscious states.

\section{Time-resolved smoothness is sufficient to explain emergent dynamics}

An important advantage of our approach is the ability to go beyond correlations and evaluate the sufficiency of basic constraints to explain emergent phenomena. We first evaluated the sufficiency of model data to explain dynamical stability, an emergent dynamical phenomenon [5]. We followed previous work to estimate dynamical stability using the eigenvalue spectrum of a time-resolved vector autoregressive model [24, 38, 65], a locally linear approximation of distributed brain activity. Theory predicts that interesting, weakly stable dynamics have largest eigenvalues with magnitudes that approach 1. Conversely, less interesting, strongly stable dynamics have largest eigenvalues with smaller magnitudes. Previous stability analyses of monkey electrocorticography recordings found that consciousness associates with weakly stable dynamics, and propofol anesthesia associates with strongly stable dynamics $[38,63,66]$.

Figure 3a-c shows that the smoothness model, and no other evaluated models, reproduced empirical changes in dynamical stability across consciousness and propofol anesthesia. We quantified dynamical stability by the median magnitude of the $5 \%$ largest eigenvalues. Stability estimates pooled across four empirical recordings showed that this index had a median [95\% uncertainty interval] of 0.995 [0.992, 0.998] during an interval of consciousness, and 0.985 [0.972, 0.993] during an interval of anesthesia ( $p<10^{-10}$, Wilcoxon rank sum test). Similarly, the stability of smoothness model data was 0.994 [0.992, 0.995] during consciousness, and $0.986[0.920,0.994]$ during anesthesia $\left(p<10^{-10}\right)$. In contrast, power-spectrum 

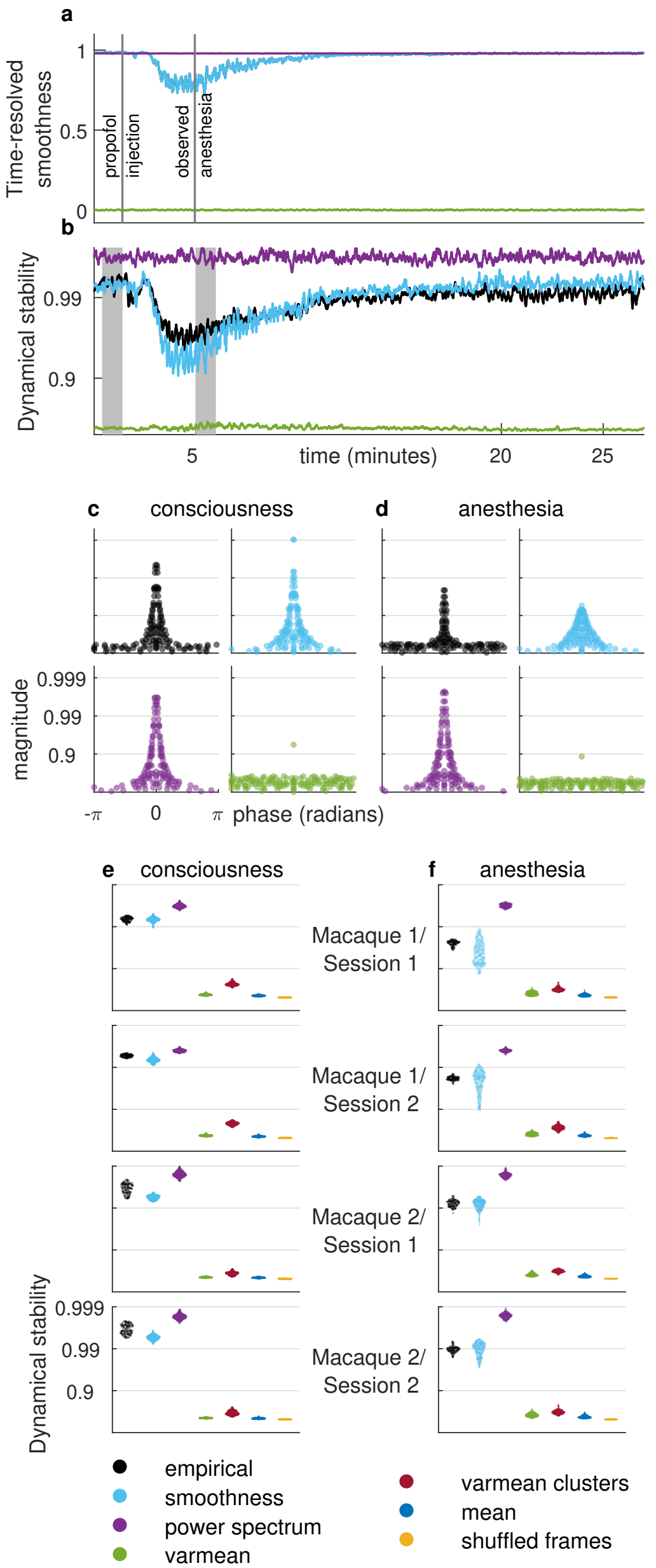

Figure 3: See caption on next page. 
Figure 3: Time-resolved smoothness explains changes in dynamical stability across consciousness and propofol anesthesia. (a) Fluctuations of time-resolved smoothness for smoothness, power-spectrum and varmean model data in a representative electrocorticography recording. (b) Dynamical stability index, the median value of the 5\% largest eigenvalues, for the same recording. Note the strong correlation of time-resolved smoothness with dynamical stability. (c-d) Phase and magnitude of eigenvalues of a vector autoregressive model fit to a single window from periods of consciousness and anesthesia (shaded intervals in panel (b), equivalent to intervals in Figure 2). Note the drop of dynamical stability in anesthesia in empirical data, as well as in smoothness model data. (e-f) Dynamical stability index across all empirical and model data, pooled over all recordings and averaged over all windows denoting periods of consciousness and anesthesia. Results on model data were derived from 50 samples for each model of each dataset. Extended Data Figures 5-8 show individual results for all empirical and model datasets.

model data showed weakly stable dynamics $(0.997$ [0.996, 0.999]) during consciousness but no change in stability $(0.998[0.996,0.999])$ during anesthesia $(p=0.243)$. All other model data showed strongly stable dynamics across consciousness and anesthesia (Figure 3e-f, Extended Data Figures 5-8).

\section{Time-resolved smoothness is sufficient to explain scale-free statistics}

We next evaluated the extent with which model data accounted for scale-free statistical phenomena. We considered two commonly studied signatures of these phenomena: $1 / f$ powerfrequency relationships and long-range temporal correlations. $1 / f$ denotes the decay of spectral power as a function of frequency. This scale-free, or arrhythmic, power-frequency relationship is distinct from neuronal oscillations, which represent narrow peaks in frequency spectra. This relationship is typically represented by the slope of the $1 / f$ power spectrum.

Figure 4a-c, and Extended Data Figures 9-19, show that, inline with previous observations $[15,35,67]$, we found robust $1 / f$ scaling of spectral power in a broad 1 to $80 \mathrm{~Hz}$ frequency range. Specifically, we found that empirical data across all intracranial EEG recordings showed a mean [95\% uncertainty interval] exponent values of 2.834 [1.827, 3.989]. Notably, smoothness model data likewise showed prominent $1 / f$ scaling with exponents 2.071 [1.869, 2.639] across all intracranial EEG recordings. The value of these exponents was necessarily more uniform because the model is spatially agnostic by definition. Nevertheless, these values 
a Human SEEG
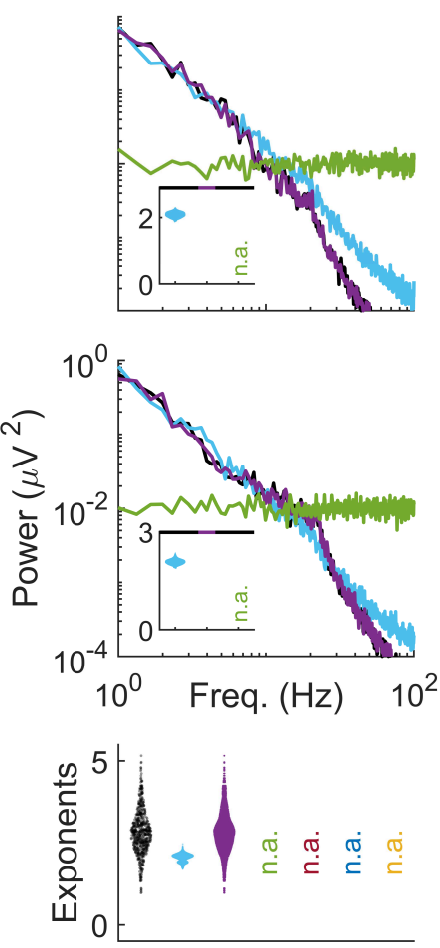

d Human SEEG
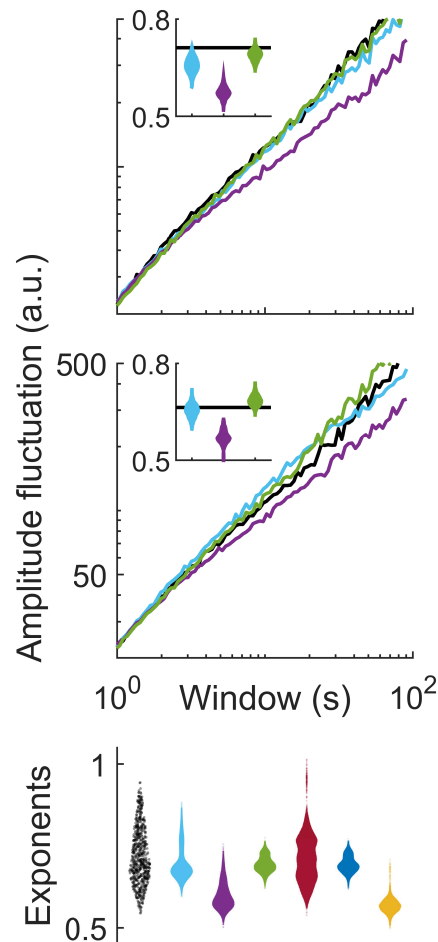

- empirical

- smoothness

- power spectrum

varmean
Human ECoG

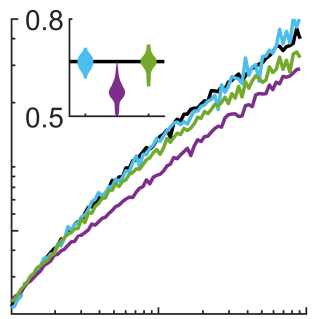

b Human ECoG
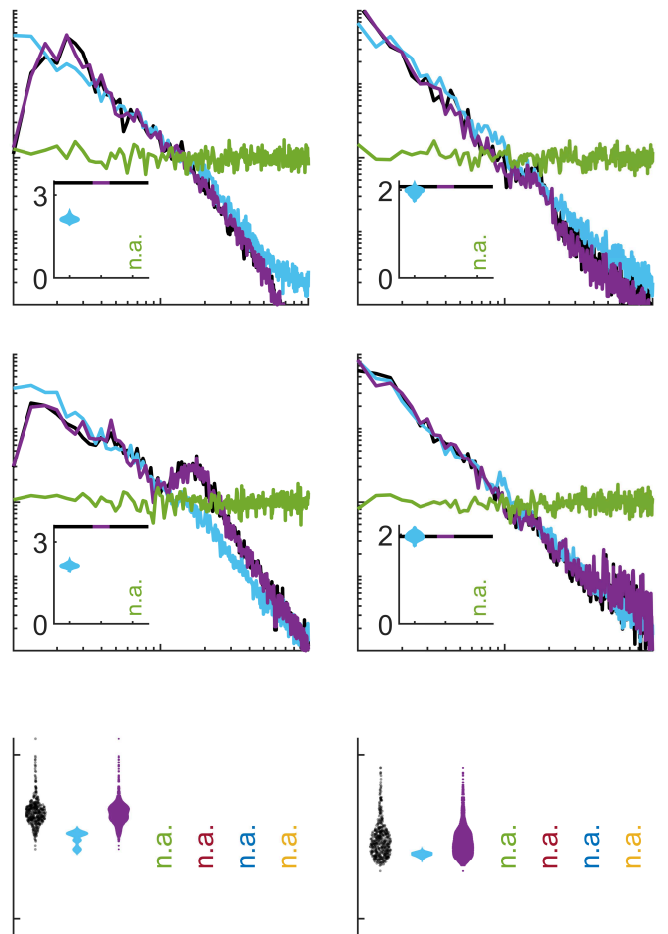

f Macaque ECoG
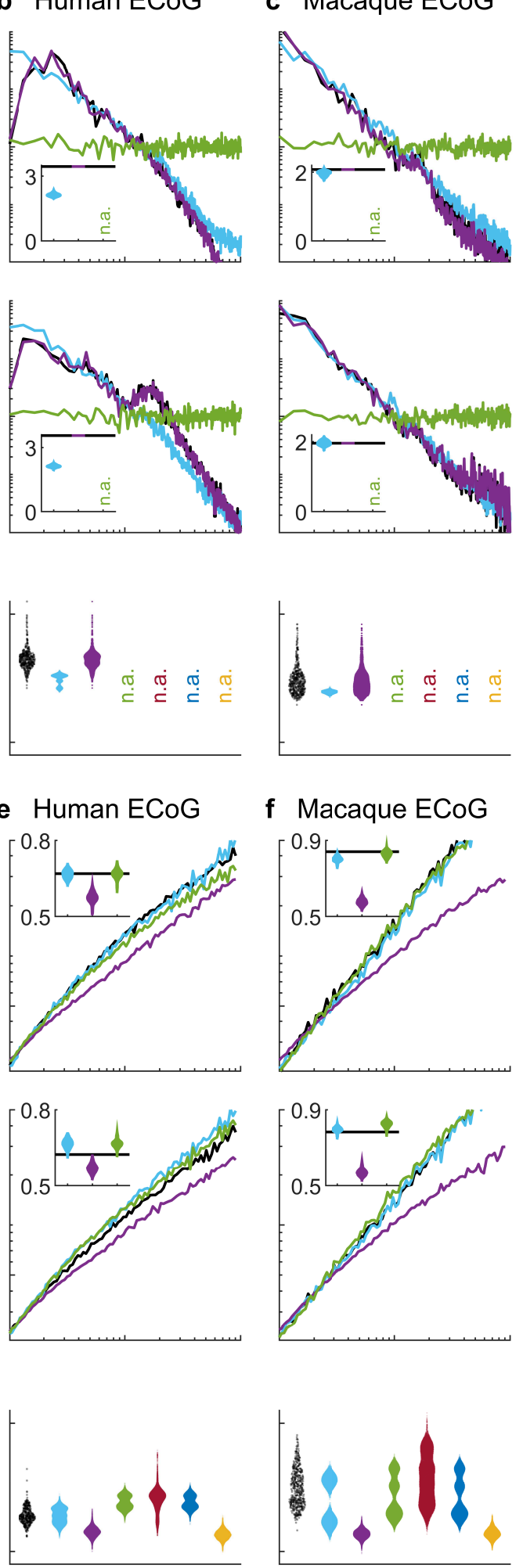
Figure 4: Time-resolved smoothness explains empirical $1 / f$ scaling and long-range temporal correlations. (a-c) Top two panels show $1 / f$ scaling of spectral power as a function of frequency for two channels of representative intracranial EEG recordings. Insets show violin plots of $1 / f$ slope exponents benchmarked against empirical exponents (solid black lines). Bottom panels show violin plots of exponents pooled across all channels and all empirical and model datasets. n.a. denotes lack of power-law fit. (d-e) Top panels show scaling of time-resolved amplitude as a function of window for two representative channels. Insets show violin plots of scaling exponents, benchmarked against empirical exponents (solid black lines). Bottom panels show violin plots of all exponents pooled across all channels and all empirical and model datasets. Results on model data were derived from 50 samples for each model of each dataset. Extended Data Figures 9-19 show individual results for all empirical and model datasets. Abbreviations: SEEG, stereotactic EEG; ECoG, electrocorticography; Ca, calcium imaging.

were inline with empirical values ( $p=0.170$ that any model exponent was at least equal to any empirical exponent). Notably, no model data, with the trivial exception of the power-spectrum model data, showed $1 / f$ scaling.

We next considered long-range temporal correlations, emergent phenomena that track the statistical self-similarity of long timeseries. Typically, these correlations are defined by the scaling exponent of mean fluctuations in the amplitude envelope in alpha or beta frequency bands, using detrended fluctuation analysis, a method especially applicable to nonstationary timeseries [68]. The scaling exponent between window sizes and mean fluctuations indexes the presence of statistical self-similarity. Values of this exponent considerably larger than 0.5 denote interesting, self-similar (fractal-like) structure. In contrast, values close to 0.5 denote less interesting, random (white-noise-like) structure [22].

Figure 4d-f, and Extended Data Figures 9-19, show robust power-law scaling for mean fluctuations in amplitude for all empirical datasets in the alpha frequency band (results were similar for the beta frequency band, not shown). The scaling exponents for empirical datasets were within the range previously reported in literature. Specifically, we found that empirical recordings across all intracranial EEG modalities showed a median [95\% uncertainty interval] exponent values of 0.687 [0.599, 0.868]. Smoothness model data showed similar exponents across all intracranial EEG recordings (0.666 [0.594, 0.800], $p=0.409)$. In contrast, powerspectrum model data showed exponents that were considerably closer to white-noise values $(0.576[0.543,0.646])$, and that significantly deviated from values of empirical exponents $(p=$ 
0.048). Collectively, these findings indicate that only the smoothness model data exhibited both $1 / f$ scaling and long-range temporal correlations across datasets.

\section{Time-resolved smoothness is sufficient to explain avalanche statistics}

Neural avalanches are transient periods of coordinated activity between groups of neurons or brain regions. The study of avalanche dynamics plays a prominent role in the theory of neural criticality. One commonly studied phenomenon in this literature is the power-law scaling of avalanche size and duration distributions. Nonetheless, studies have also emphasized that this scaling may not be strongly indicative of critical dynamics [69], and recent work has focused on more specific signatures of criticality, including power-law relationships between size and duration exponents [70], as well as shape collapse, a universal scaling relationship of avalanche phase with activity $[37,70,71]$. Shape collapse, in particular, is a strict criterion of neural criticality, obtained through averaging hundreds or thousands of individual avalanches. Theory predicts that at criticality, collapsed temporal profiles of avalanches will converge to a universal shape independent of avalanche duration $[28,70,71]$.

We first evaluated the presence of avalanche phenomena in all intracranial EEG datasets. Figure 5, and Extended Data Figures 20-30, show robust signatures of power-law scaling for avalanche sizes, durations, and size-duration relationships. Specifically, across all intracranial EEG datasets, avalanche sizes decayed with a median [95\% uncertainty interval] power-law exponents of 1.340 [1.221, 1.486]. Correspondingly, avalanche durations decayed with an exponent of 1.355 [1.043, 1.585]. Finally, avalanche sizes scaled as a function of avalanche durations with exponents of 1.539 [1.237, 1.960]. Smoothness model data largely recapitulated these signatures with exponents of $1.285[1.150,1.430](p=0.260)$ for avalanche sizes, 1.280 $[1.000,1.520](p=0.384)$ for avalanche durations, and $1.584[1.444,1.906](p=0.606)$ for scaling of size as a function of duration. In contrast, all other model data, except powerspectrum model data, had a considerable number of exponents that significantly deviated from empirical values (Figure 5).

Figure 6, and Extended Data Figures 20-30, show that empirical data exhibited avalanche shape collapses indicative of universal scaling. Notably, smoothness, power-spectrum, and 
a Human SEEG
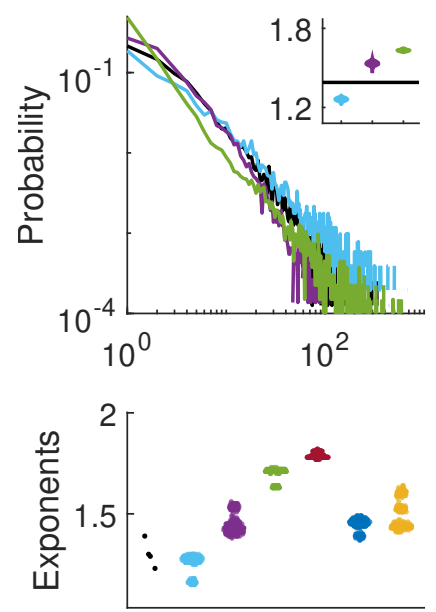

e Human SEEG
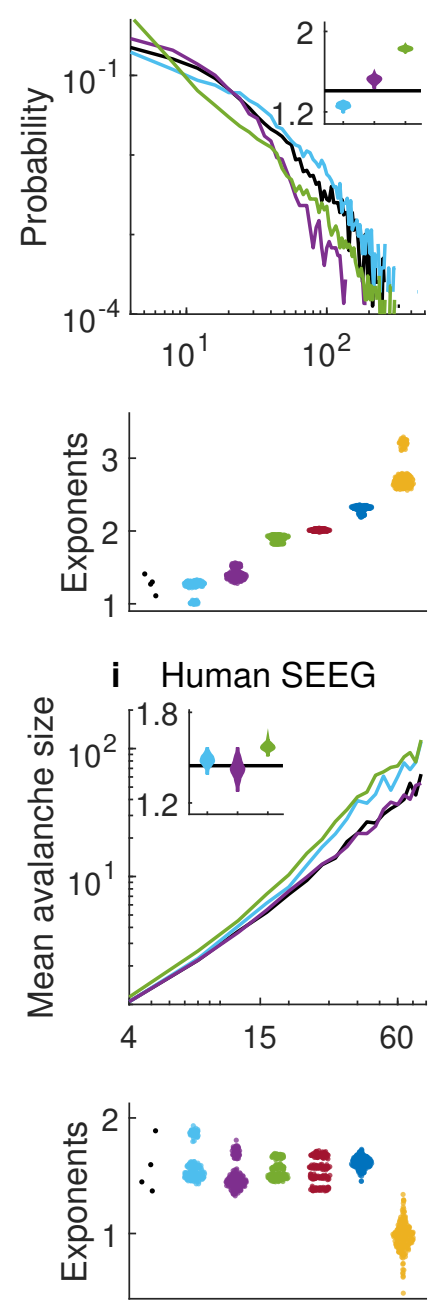

b Human ECoG

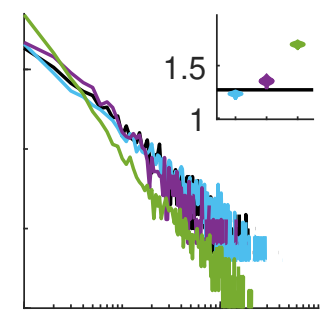

c Monkey ECoG

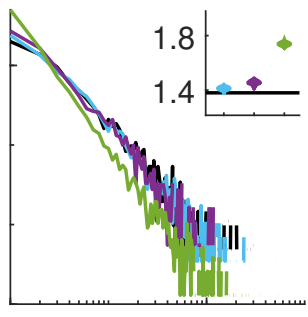

Avalanche size (no. activations)

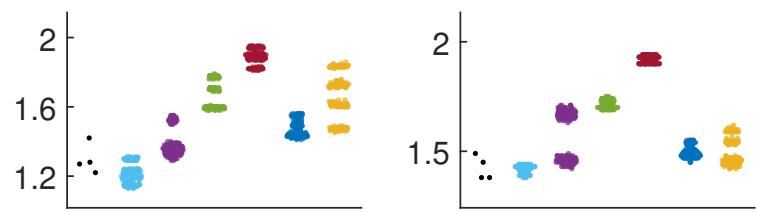

f Human ECoG

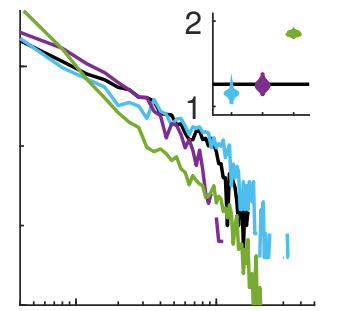

Avalanche duration (ms)
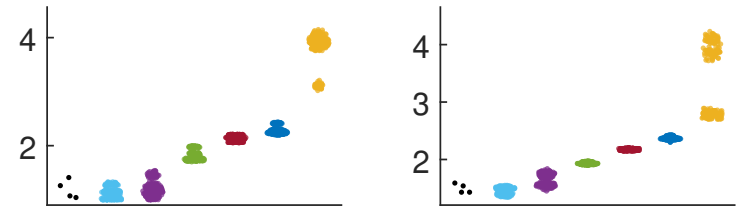

j Human ECoG

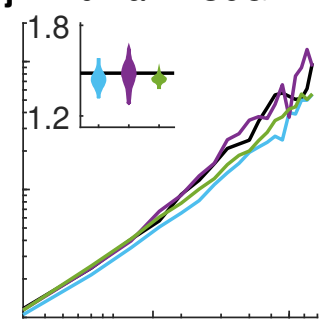

k Monkey ECoG

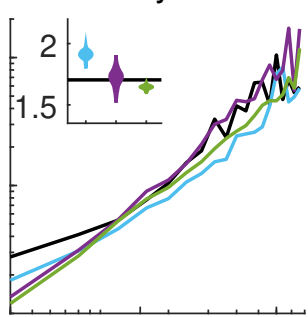

Avalanche duration (ms)
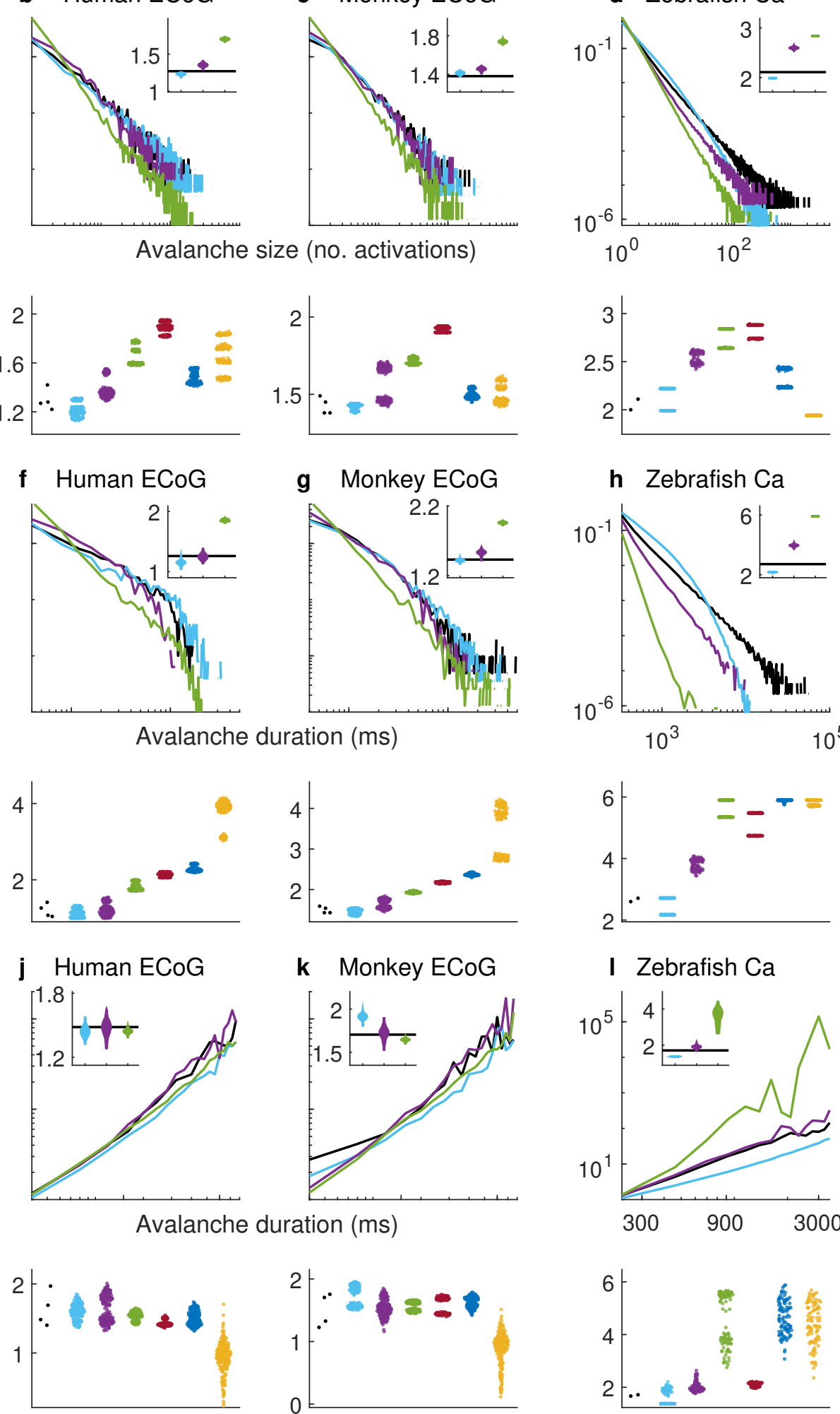

h Zebrafish $\mathrm{Ca}$
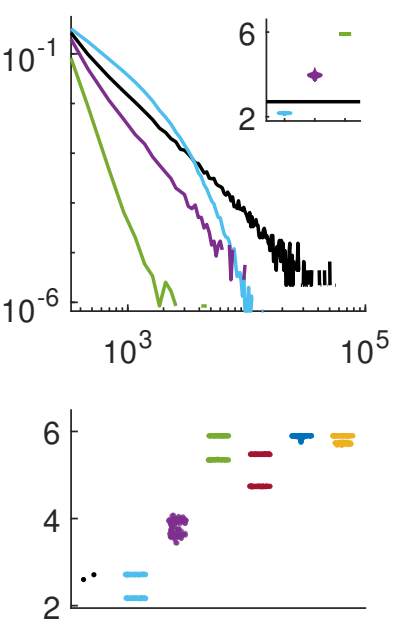

I Zebrafish $\mathrm{Ca}$

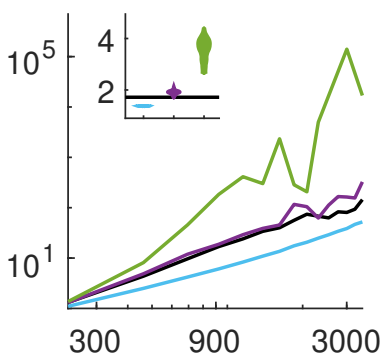

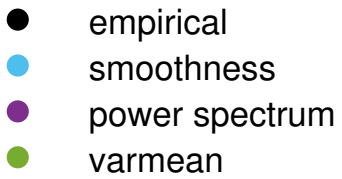

- $\quad$ marmean clusters
- $\quad$ shuffled frames

Figure 5: See caption on next page. 
Figure 5: Time-resolved smoothness explains avalanche statistics. (a-d) Avalanche size probability distributions. (e-h) Avalanche duration probability distributions. (i-l) Scaling of avalanche size and duration exponents. Inset violin plots show normalized distributions of model exponents, benchmarked against empirical exponents (solid black lines). Bottom panels show violin plots of all empirical and model exponents pooled across all empirical and model datasets. Results on model data were derived from 50 samples for each model of each dataset. Extended Data Figures 20-32 show individual results for all empirical and model datasets. Abbreviations: SEEG, stereotactic EEG; ECoG, electrocorticography; Ca, calcium imaging.

varmean model data all had similar shape collapses. The mean avalanche temporal profiles in these model data all peaked at similar times. Indeed, the variance between rescaled avalanche shapes had a median [95\% uncertainty interval] of 0.098 [0.037, 0.299] for empirical data, $0.088[0.043,0.349](p=0.507)$ for smoothness model data, 0.080 [0.043 0.204] $(p=0.558)$ for power-spectrum model data, and 0.120 [0.055, 0.419] $(p=0.383)$ for varmean model data. All other models lacked universal shape collapses. These results imply that basic timeresolved constraints are sufficient to generate data that pass stringent tests of criticality.

\section{Time-resolved smoothness of brain-wide calcium imaging data}

Finally, we sought to evaluate emergent phenomena in calcium imaging recordings of fictively swimming zebrafish. These data provide an unmatched spatiotemporal resolution of brainwide cellular activity [55]. At the same time, the distinct nature of these data present several methodological challenges (Table 1). Specifically, in contrast to intracranial EEG recordings, brain-wide calcium imaging recordings were imaged at a relatively low frequency (about 3Hz), had many more nodes (about 100 thousand neurons), and many fewer timepoints (about 6 thousand). The low sampling rate limited our evaluation to critical emergent phenomena, inline with recent work [37]. The large number of nodes imposed memory requirements which precluded the use of nullspace sampling and required the adoption of memory efficient but slow constrained randomization methods [48, 49]. Moreover, low-frequency imaging resulted in considerably lower values of time-resolved smoothness (Figure 1d). We compensated for this drop in smoothness by constraining time-resolved smoothness between a single frame and its four adjacent neighbors. Similarly, the distinct dimensionality of these data resulted in varmean-clusters models that had substantially more constraints. Finally, standard avalanche- 


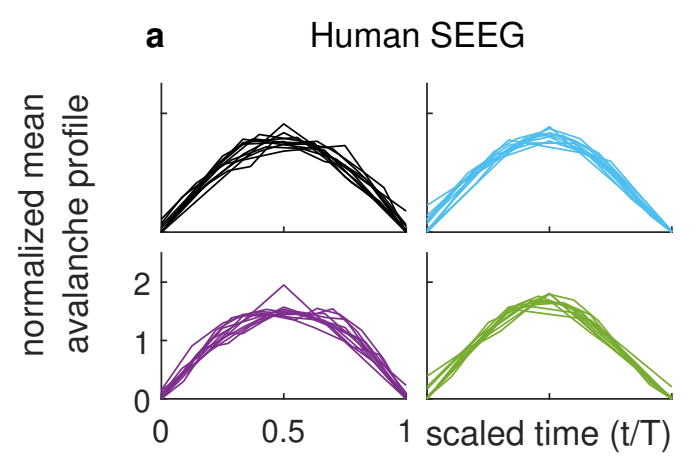

b Human ECoG
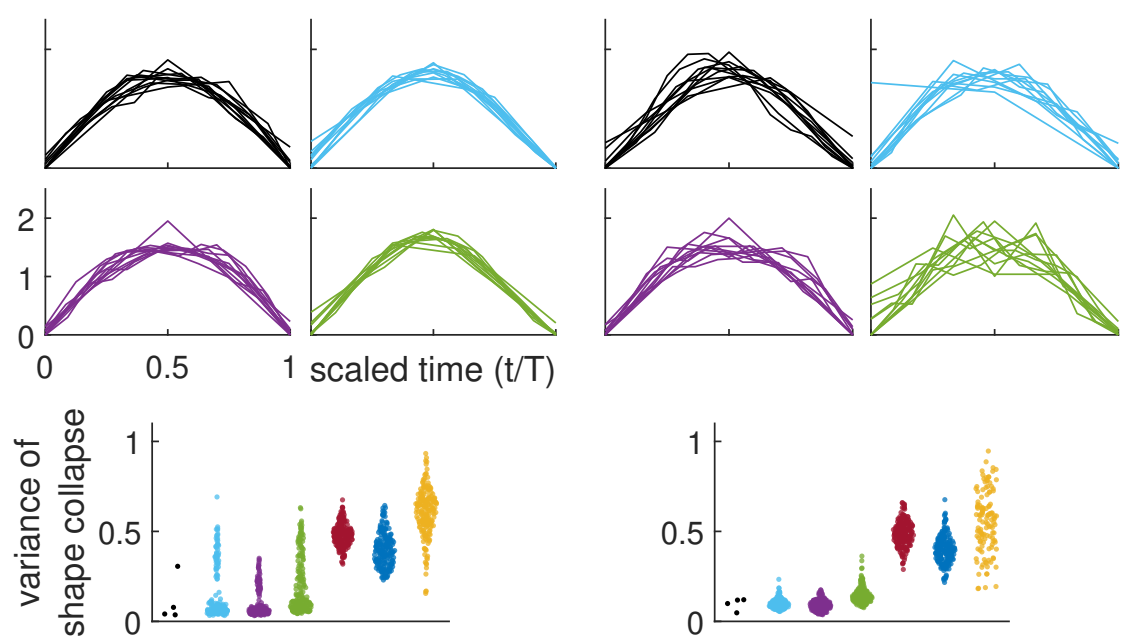

C

Monkey ECoG
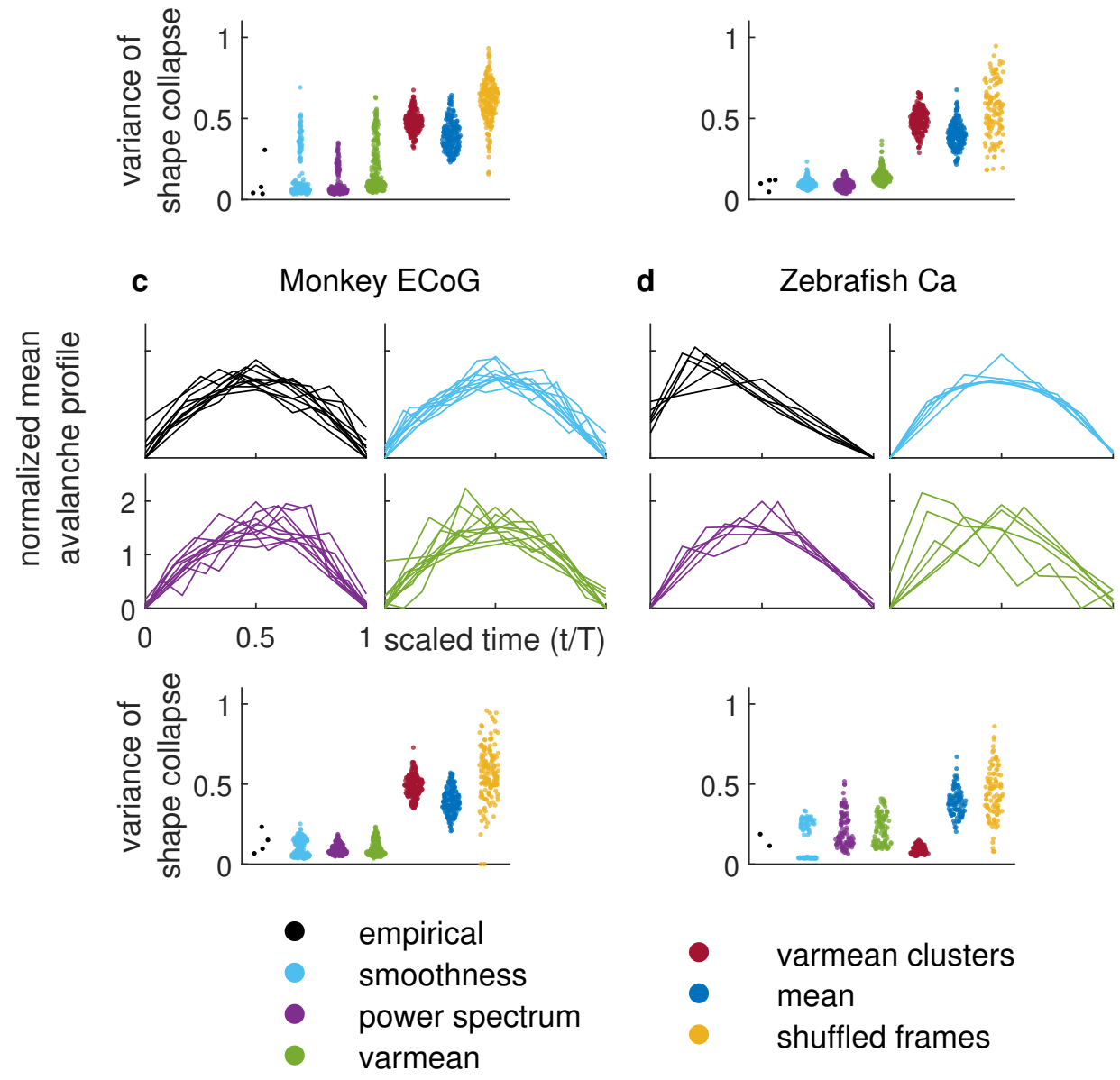

- varmean clusters

mean

- shuffled frames

Figure 6: Model data constrained by time-resolved features pass stringent tests of criticality. (a-d) Shape collapses: scaling of mean temporal profiles with avalanche activity. Bottom panels show violin plots of all empirical and model variance between avalanche scaling profiles (lower is better). Results on model data were derived from 50 samples for each model of each dataset. Extended Data Figures 20-32 show individual results for all empirical and model datasets. Abbreviations: SEEG, stereotactic EEG; ECoG, electrocorticography; Ca, calcium imaging. 
detection methods in these data result in a trivial detection of a single never-ending brain-wide avalanche. We compensated for this problem by adopting a spatially contiguous definition of avalanches [37], which in turn penalizes spatially agnostic models, such as the smoothness model.

These caveats aside, our modeling results on these datasets were generally inline with results on intracranial EEG data. Figure 6, and Extended Data Figures 31-32 show that empirical calcium-imaging avalanche sizes decayed as power laws with exponents 2.110 and 2.000, avalanche durations decayed as power laws with exponents 2.710 and 2.600 , and avalanche sizes scaled as a function of durations with power-law exponents of 1.717 and 1.667. Smoothness model data had similar median [95\% uncertainty interval] values of these exponents. Specifically, it had avalanche size exponents of 2.105 [1.999, 2.220], duration exponents of 2.445 [2.160, 2.720], and size-duration scaling exponents of 1.523 [1.374, 2.038] (we do not report $\mathrm{p}$ values because we only have two observations of empirical exponents). The exponents of most other models deviated to a larger extent from those values, while shape collapse results were largely inline with those observed on intracranial EEG datasets (see Figure 6, and Extended Data Figures 31-32 for a summary of all results).

\section{Discussion}

We developed a scalable method to generate model data constrained by interpretable empirical properties in large neurophysiological recordings. We used this method to evaluate the extent with which empirical constraints explained major statistical and dynamical emergent phenomena across species and modalities. Our results showed that a simple model constrained by empirical time-resolved smoothness, variance, and mean, recapitulated all evaluated emergent phenomena across modalities and species. This model outperformed all other evaluated models, including a model constrained by power spectra and cross-correlation structure.

The nature of time-resolved smoothness offers two strong clues to the nature of our evaluated emergent neural phenomena. First, we know that time-resolved smoothness is agnostic to properties of individual nodes, and therefore cannot, by definition, capture mechanistic network interactions. Put another way, knowledge of time-resolved smoothness tells us little 
about ways by which specific network interactions give rise to cognition and behavior. On the other hand, time-resolved smoothness robustly tracks spatially non-specific changes in distributed activity. It follows that the sufficiency of this property to explain emergent neural phenomena implies that such emergent phenomena likely do not capture functional mechanisms, but instead may reflect spatially non-specific modulation of these mechanisms $[72,73]$.

Our results offer to unify, simplify, and reinterpret theoretical frameworks in systems neuroscience in several ways. First, these results offer a roadmap for future neurobiological explanations of emergent phenomena, through discovery of molecular, cellular or circuit mechanisms that underpin time-resolved smoothness. Prime candidates for such mechanisms include slow neuromodulation of cortical excitability and arousal by widely projecting subcortical systems [74-76]. Second, our results allow to reinterpret the notion of cortical timescales [77-80]. Specifically, we propose that these timescales may primarily reflect regional contributions to time-resolved smoothness of distributed activity. In turn, this intuitive interpretation naturally de-emphasizes the need to explain the functional significance of scale-free structure of regional activity. Third, our results can substantially simplify the large translational literature centered on emergent phenomena [32,33]. Much of this literature focuses on changes in diverse phenomena across clinical states [37-39]. Our findings show that a single marker of brain activity can replace, and may serve as a final common pathway, for many often disparate signatures of emergent activity. In this way, these findings promise to reduce redundant explanations and accelerate translational progress.

Finally, we emphasize that our study was ultimately made possible by an unbiased and efficient method of surrogate data. As neuroscientists generate increasingly bigger datasets, it becomes increasingly important to extract insights from these data with scalable and interpretable methods $[48,49]$. We hope that future adoption of our sampling method will help with this endeavor and thus enable more unified and cohesive investigations of distributed brain activity. 


\section{Methods}

\section{Generation of empirically constrained model data}

\section{Definition of time-resolved smoothness}

We defined time-resolved smoothness as,

$$
\operatorname{smoothness~}_{i, \text { lag }}=\frac{\sum_{j=1}^{n}\left(\mathbf{v}_{i}^{j}-\overline{\mathbf{v}}_{i}\right)\left(\mathbf{v}_{i+\text { lag }}^{j}-\overline{\mathbf{v}}_{i+\text { lag }}\right)}{\sqrt{\sum_{j=1}^{n}\left(\mathbf{v}_{i}^{j}-\overline{\mathbf{v}}_{i}\right) \sum_{j=1}^{n}\left(\mathbf{v}_{i+\mathrm{lag}}^{j}-\overline{\mathbf{v}}_{i+\mathrm{lag}}\right)}}
$$

where $\mathbf{v}_{i}^{j}$ denotes the activity of node $j$ at time $i, \overline{\mathbf{v}}_{i}$ denotes the mean activity of all nodes at time $i$, and lag is an integer.

It is easy to show that average time-resolved smoothness is related to average node autocorrelation as follows,

$$
\left(\begin{array}{c}
\text { time-resolved smoothness } \\
\text { averaged over all timepoints }
\end{array}\right) \approx\left(\begin{array}{c}
\text { node autocorrelation } \\
\text { averaged over all nodes }
\end{array}\right)
$$

This equivalence holds because both quantities in equation 1 represent averaged dot products, normalized either over the activity of all nodes at a single timepoint (time-resolved smoothness), or over the activity of a single node at all timepoints (node autocorrelation). At the same time, the non-averaged versions of these quantities are incommensurate because time-resolved smoothness is defined for each timepoint, while autocorrelation is defined for each node.

\section{General formulation of nullspace sampling}

Our sampling method expresses empirical features of interest as sets of linear equations. Formally, let us define $\mathbf{v} \in \mathcal{R}^{n \times t}$ to be the empirical timeseries of $n$ nodes and $t$ timepoints. Let us also define $\operatorname{vec}(\cdot)$ to be a vectorization or flattening operator, such that $\operatorname{vec}(\mathbf{v}) \in \mathcal{R}^{n t \times 1}$.

Let us now consider $m$ features of interest, define $\mathbf{b} \in \mathcal{R}^{m \times 1}$ to be the empirical values of these features, and define $\mathbf{A} \in \mathcal{R}^{m \times n t}$ to represent the coefficient matrix for these features. 
Formally we can write,

$$
\mathbf{A} \operatorname{vec}(\mathbf{v})=\mathbf{b}
$$

Without loss of generality, let us assume that the empirical features of interest are linearly independent (and therefore that the matrix $\mathbf{A}$ is full rank). Since the number of these features will, in most cases, be much smaller than the number of data elements ( $m \ll n t$ ), we end up with an underdetermined set of linear equations. It follows that we can generate model data by unbiasedly sampling from the solution space of this set.

Linear algebra tells us that this solution space is spanned by orthogonal unit vectors that form the nullspace of the coefficient matrix $\mathbf{A}$ [81]. Let us define $\mathbf{Z} \in \mathcal{R}^{n t \times(n t-m)}$ as this nullspace, such that $\mathbf{A Z}=0$. We can then express the solution space as,

$$
\operatorname{vec}(\mathbf{x})=\operatorname{vec}\left(\mathbf{x}_{0}\right)+k \mathbf{Z} \mathbf{q}
$$

where $x_{0}$ denotes the minimum-norm solution, $k$ is a scaling constant and $\mathbf{q} \in \mathcal{R}^{(n t-m) \times 1}$ is a uniformly sampled random weighting vector. Geometrically, each row in A represents an unbounded hyperplane in $n t$ dimensions and the solution space is an $n t-m$ vector space formed by the intersection of these hyperplanes.

We regularized the space of these solutions by constraining the Frobenius norm of all model timeseries to empirical values. We did this by first setting the norm of $\mathbf{q}$ to 1 . Uniformly sampling $\mathbf{q}$ with $|\mathbf{q}|=1$ is equivalent to sampling from the surface of the $n t-m$ dimensional hypersphere of radius 1 and centered at the origin. This sampling can be achieved by generating vectors from the $n t-m$ dimensional standard normal distribution and rescaling the vectors to have unit norm. This procedure guarantees to produce random samples uniformly distributed on the surface of the hypersphere [82]. We then used the scaling parameter $k$ to rescale the norm to match the empirical timeseries. To determine the value of $k$, we note that $\operatorname{vec}\left(\mathbf{x}_{0}\right)=\mathbf{A}^{\dagger} \mathbf{b}$ where $\cdot^{\dagger}$ denotes the Moore-Penrose pseudoinverse. From the standard theorem of algebra, we know that $\operatorname{vec}\left(\mathbf{x}_{0}\right)$ is always orthogonal to $\mathbf{Z}$. Because the 
two additive components in equation 3 are orthogonal, we can use Pythagoras theorem to set $k=\sqrt{|\mathbf{v}|^{2}-\left|\mathbf{x}_{0}\right|^{2}}$ and thereby ensure that the Frobenius norm of $\mathbf{x}$ equals the norm of $\mathbf{v}$.

Once we set $k$ and $\mathbf{q}$, we can then directly sample datasets $\mathbf{x}$. Because $\mathbf{Z}$ is an orthonormal matrix, our sampling is guaranteed to be uniform as long as $\mathbf{q}$ is drawn from a multivariate uniform distribution. We used standard MATLAB functions such as qr and svd to build orthonormal nullspaces. Once we compute the nullspace, it becomes trivial to sample $q$ from a multivariate uniform distribution and generate model timeseries. Box 1 summarizes the complete sequence of steps in the general formulation of nullspace sampling.

Box 1. Summary of nullspace sampling

1. Define empirical features through linear equations, $\mathbf{A} \operatorname{vec}(\mathbf{v})=\mathbf{b}$.

2. Compute orthonormal nullspace matrix $\mathbf{Z}$ of the coefficient matrix $\mathbf{A}$.

3. Compute the minimum-norm solution vec $\left(\mathrm{x}_{0}\right)$.

4. Uniformly sample the weighting vector $\mathbf{q}$ and compute the scaling parameter $k$.

5. Generate model data using $\operatorname{vec}(\mathbf{x})=\operatorname{vec}\left(\mathbf{x}_{0}\right)+k \mathbf{Z q}$.

\section{Sequential nullspace sampling with nonlinear constraints}

The general formulation of nullspace sampling admits only linear constraints, and produces massive nullspace matrices of close to $(n t)^{2}$ elements. This formulation is of limited use for constraining time-resolved smoothness and other nonlinear constraints in large datasets. Nonetheless, here we exploited the sequential definition of time-resolved smoothness to allow nullspace sampling with nonlinear constraints in long neurophysiological recordings.

We first expressed empirical timeseries $\mathbf{v}$ as a sequence of vectors $\mathbf{v}_{1}, \mathbf{v}_{2}, \ldots \mathbf{v}_{t}$, where $\mathbf{v}_{i} \in \mathcal{R}^{n \times 1}$ denotes the activity of all nodes at time $i$. Likewise, we expressed model timeseries $\mathbf{x} \in \mathcal{R}^{n \times t}$ as a sequence of activity vectors $\mathbf{x}_{1}, \mathbf{x}_{2}, \ldots \mathbf{x}_{t}$, with $\mathbf{x}_{i} \in \mathcal{R}^{n \times 1}$. We then defined correlations between adjacent timepoints as $c_{1}, c_{2}, \ldots c_{t-1}$, where $c_{i}=\mathbf{v}_{i} \cdot \mathbf{v}_{i+1}=$ $\mathbf{x}_{i} \cdot \mathbf{x}_{i+1}$ and $\cdot$ denotes the dot product. 
This formulation allowed us to generate model timeseries with correlation constraints sequentially, timepoint by timepoint. Specifically, at each timepoint $i$, we used model data $\mathbf{x}_{i}$ as the coefficient matrix to generate model data at timepoint $i+1$. Without loss of generality, we used the following algorithm to generate $\mathbf{x}_{i+1}$ with zero mean and unit standard deviation:

1. For $i=0$, sample a random normally distributed vector $\mathbf{x}_{1}$ with zero mean and unit standard deviation.

2. For $i=1,2 \ldots t-1$, sample $\mathbf{x}_{i+1}$ by solving,

$$
\begin{aligned}
{\left[\mathbf{x}_{i}, \mathbf{1}\right]^{\top} \mathbf{x}_{i+1} } & =\left[\begin{array}{l} 
\\
c_{i} \\
0
\end{array}\right] \\
\left|\mathbf{x}_{i+1}\right| & =n-1
\end{aligned}
$$

where 1 denotes the all-ones vector. The structure of the coefficient matrix in equation 4 guarantees that $\mathbf{x}_{i+1}$ will always have zero mean, and together with equation 5 guarantees that the vector will have unit standard deviation. Model vectors can then be simply rescaled to match the mean and standard deviation of empirical data.

In practice, this sequential generation of empirical data required nullspace matrices with approximately $n^{2}$ elements, and scaled linearly with the number of timepoints $t$. This approach was feasible for long intracranial EEG data $(n \approx 100)$, where it outperformed standard constrained randomization methods $[48,49]$ by orders of magnitude. On the other, the approach was not feasible for brain-wide cellular calcium imaging datasets $\left(n \approx 10^{5}\right.$ ). For these data, we replaced nullspace sampling with memory-efficient but slow constrained randomization methods.

\section{Implementation of mean and variance constraints}

In some cases (mean, varmean, and varmean-clusters models), we constrained model data by empirical mean and variance, but not by smoothness. In such cases, we generated $\mathrm{x}_{i}$ by 
solving

$$
\begin{aligned}
\mathbf{1}^{\top} \mathbf{x}_{i} & =0 \\
\text { with } \quad\left|\mathbf{x}_{i}\right| & =n-1 .
\end{aligned}
$$

To enforce these constraints within spatiotemporal clusters (varmean-clusters model), we used k-means clustering to group $n$ nodes into spatial clusters, and separately $t$ timepoints into temporal clusters. For long neurophysiological recordings, we set spatial clusters to have on average 5 nodes, and separately temporal clusters to have an average of 50 timepoints. To prevent an excessive number of clusters in brain-wide cellular calcium imaging recordings, we set spatial clusters in these datasets to have an average of 250 nodes, and temporal clusters to have an average of 100 timepoints.

To further optimize the efficiency of nullspace sampling, we derived an analytical expression for the nullspace $\mathbf{Z} \in \mathcal{R}^{n \times(n-1)}$ of the coefficient matrix $\mathbf{A}=\mathbf{1}^{\top}$ in equation 6 ,

$$
\mathbf{Z}=\left(\begin{array}{ccccc}
-c & -c & -c & \ldots & -c \\
b & -a & -a & \ldots & -a \\
-a & b & -a & \ldots & -a \\
-a & -a & b & \ldots & -a \\
\vdots & \vdots & \vdots & \vdots & \vdots \\
-a & -a & \ldots & -a & b
\end{array}\right),
$$

where $a, b$ and $c$ denote solutions to the following set of equations:

$$
\begin{aligned}
b-a(n-2)-c & =0 \\
c^{2}+a^{2}(n-2)+b^{2}-1 & =0 \\
c^{2}-2 a b+(n-3) a^{2} & =0
\end{aligned}
$$


One can directly verify that this solution is an orthonormal nullspace of the coefficient matrix $\mathbf{A}=\mathbf{1}^{\top}$, such that $\mathbf{Z}^{\top} \mathbf{Z}=\mathbf{I}$ and $\mathbf{A Z}=0$. In practice, this analytical formulation allowed us to eschew the use of numerical computational methods for computing the nullspace of mean, varmean, and varmean-clusters models.

\section{Phase randomization}

We generated phase-randomized model data using a well-known algorithm from the physics and neuroscience literature $[56,57]$. Briefly, we first used the Fourier transform to compute the phase and amplitude of each timeseries. We then rotated the phase of all nodes at each frequency by a random complex variable $e^{i \phi}$ with $\phi$ uniformly distributed in $[-\pi, \pi]$. Finally, we obtained model timeseries by computing the inverse Fourier transform of these phaserandomized data. This method is guaranteed to generate maximally random data that preserve nodal power spectra and full cross-correlation structure.

\section{Details of data acquisition}

\section{Macaque electrocorticography (Neurotycho)}

Data were collected at the Laboratory for Adaptive Intelligence, Brain Science Institute, RIKEN. Electrocorticography recordings were made from two male macaques before, during, and after administration of propofol anesthesia. Grid electrodes were implanted on the frontal, parietal, temporal, and occipital lobes. Additional experimental details for these data are provided in previous studies $[50,59]$. These data are publicly available from http://www.neurotycho.org.

\section{Human electrocorticography}

Data were acquired at the University of California Irvine Hospital. Electrocorticography recordings were made from adult epileptic patients performing a visuospatial working memory task. Grid electrodes were implanted in frontal and medial temporal lobes. Additional experimental details for these the data are provided in a previous study [51]. These data are publicly available from http://www.crcns.org [83]. 


\section{Human stereotactic EEG}

Data were acquired at Vanderbilt University Medical Center. Stereotactic EEG recordings were made from adult epilepsy patients, one day after lead implantation and before medication wean. The patients were instructed to keep their eyes closed and remain awake for twenty minutes. Electrodes were implanted in cortical regions, depending on suspected seizure origin. Additional experimental details for these data are provided in a previous study [52].

\section{Zebrafish calcium imaging}

Data were acquired at Janelia Research Campus, Howard Hughes Medical Institute. Lightsheet calcium imaging recordings were made from fictively swimming larval zebrafish embedded in agarose. The fish swam against a fixed-velocity one-dimensional moving stripe pattern. The imaging spanned almost all brain neurons expressing a genetically encoded calcium indicator (GCaMP6f). Additional experimental details for these data are provided in a previous study [53].

\section{Details of data preprocessing}

\section{Intracranical EEG}

Dynamical stability analyses were performed on recordings sampled at $1000 \mathrm{~Hz}$, in accordance with previous work $[38,63]$. All other analyses were performed on intracranial EEG data downsampled to $250 \mathrm{~Hz}$. All our results were robust to analysis and modeling of data at $1000 \mathrm{~Hz}$, but this higher sampling rate incurred unnecessary computational cost.

All recordings were highpass filtered with a cutoff of $0.5 \mathrm{~Hz}$ using the bandpassfilter function from Fieldtrip toolbox [84]. In addition, macaque electrocorticography data were notch-filtered at $50 \mathrm{~Hz}$ and $100 \mathrm{~Hz}$ to remove line noise. Likewise, human stereotactic EEG and electrocorticography data were notch-filtered at $60 \mathrm{~Hz}$ and $120 \mathrm{~Hz}$ to remove line noise. 


\section{Calcium imaging}

All images were motion corrected, and cells were segmented in contiguous and overlapping three-dimensional blocks, using non-negative matrix factorization with sparseness constraints. The resulting demixed and denoised cell segmentations showed high signal-to-noise ratio relative to raw pixel timeseries. Additional details of segmentation were provided in a previous study [53]. Our segmentation software is publicly available from https://github.com/mikarubi/voluseg

\section{Details of data analysis}

\section{Dynamical stability analysis}

We performed linearized dynamical stability analysis within temporally local time windows. We first divided the timeseries $\mathbf{v} \in \mathcal{R}^{n \times t}$, into non-overlapping windows of length $\Delta t=$ $500 \mathrm{~ms}$ [38]. We then fitted a time-invariant vector autoregressive model, representing a linear dynamical system, separately for each window $\mathbf{v}_{\mathbf{k}} \in \mathcal{R}^{n \times \Delta t}$, where $\mathbf{k}$ is a vector of timepoints within a window. The vector autoregressive model is defined as,

$$
\mathbf{v}_{\mathbf{k}+1}=\mathbf{A}_{\mathbf{k}} \mathbf{v}_{\mathbf{k}}
$$

For each window, we first estimated the system matrix $\mathbf{A}_{\mathbf{k}} \in \mathcal{R}^{n \times n}$ using least squares [85], and then computed its eigenvalues, $\mathbf{z}_{\mathbf{k}} \in \mathcal{C}^{n \times 1}$. These eigenvalues tracked the dynamical stability of this system. Specifically, the presence of all eigenvalues strictly inside the complex unit circle $\left(\left|\mathbf{z}_{\mathbf{k}}^{j}\right|<1\right.$, for $\left.j=1,2, \ldots n\right)$ implies that the system is asymptotically stable, such that external perturbations will decay exponentially. In contrast, the presence of some eigenvalues outside the complex unit circle $\left(\left|\mathbf{z}_{\mathbf{k}}^{j}\right|>1\right)$ implies that the system is unstable and that external perturbations will grow exponentially. Finally, the presence of eigenvalues on the complex unit circle $\left(\left|\mathbf{z}_{\mathbf{k}}^{j}\right| \approx 1\right)$, implies that the system is weakly stable and external perturbations will produce diverse responses [38]. 


\section{Power spectral analysis}

We used Welch's method to characterize the power spectral density over a relatively large frequency range between 1 and $80 \mathrm{~Hz}$. In brief, power spectra were computed for each channel separately, using windowed timeseries convolved with Hanning windows of length 30 seconds and then averaged over all windows.

There has been significant recent progress in the development of methods for estimating slopes of neural power spectra. Unlike earlier methods, such as coarse grained spectral analysis [15], which were strictly applicable only to completely aperiodic signals [86], more recent methods, such as IRASA [86] and Fitting Oscillations and One Over f (FOOOF) [16], are able to estimate both periodic and aperiodic signal components.

Here, we adopted FOOOF to estimate the spectral exponent of empirical and model timeseries. Formally the algorithm estimates,

$$
P(f)=\frac{10^{f_{\text {offset }}}}{\left(f+f_{\text {knee }}\right)^{e}}
$$

where $P$ is the power spectrum, $f$ is the frequency of interest, $e$ is the scaling exponent, $f_{\text {offset }}$ is the offset frequency, and $f_{\text {knee }}$ is the knee frequency [16]. More details about the application of this method to the study of neural spectra is provided in a recent study [87]. In this study, we set the maximum number of peaks to 6 , and the minimum peak height to 0.15 .

\section{Long range temporal correlation analysis}

Following initial preprossessing, we bandpass filtered the data in the alpha $(8-12 \mathrm{~Hz})$ and beta (20-30Hz) frequency bands, and applied the Hilbert transform to extract time-resolved amplitude at each electrode. We then used detrended fluctuation analysis to estimate amplitude scaling coefficients from these time-resolved amplitude timeseries [68]. Detrended fluctuation analysis is especially suitable for analysis of neurophysiological timeseries because it is unaffected by nonstationary structure. In practice, given a timeseries $\mathbf{y}^{j} \in \mathcal{R}^{1 \times t}$, we first defined 
the cumulative sum $\mathbf{Y}^{j}$ as follows,

$$
\mathbf{Y}_{i}^{j}=\sum_{i=1}^{t}\left(\mathbf{y}_{i}^{j}-t \overline{\mathbf{y}}^{j}\right)
$$

where $\overline{\mathbf{y}}^{j}$ denotes the mean activity of node $j$. We then segmented the timeseries into nonoverlapping segments of varying lengths $\Delta t$. We considered a broad range of temporal windows, from 1 second to 3 minutes, in order to robustly capture scaling behavior across multiple timescales.

For each timescale $\Delta t$, we computed $F(\Delta t)$, the root-mean-square of the detrended cumulative signal $\mathbf{Y}^{j}$ within each segment, averaged over segments. Finally, we fitted a power law of the form $F(\Delta \mathrm{t})=h \Delta t^{\alpha}$. The value of the exponent $\alpha$ denotes the presence or absence of long-range temporal correlations. A value between 0.5 and 1.0 is indicative of long range temporal correlations, while values close to 0.5 denote an uncorrelated process.

\section{Avalanche analysis}

Avalanche detection in electrophysiological datasets. Inline with previous studies, we binarized electrophysiological imaging datasets by setting to 1 deflections in local field potentials that exceeded three standard deviations below the mean $[65,88,89]$. We then detected, at each timepoint, bursts of temporally contiguous coordinated activity in these binarized timeseries. Specifically, a new avalanche is instantiated if at least one of the constituent elements becomes active. The avalanche propagates until none of the constituent elements are active. The size of an avalanche is defined by the number of active constituent elements, and the duration of an avalanche is defined by the period of its activity.

Avalanche detection in calcium imaging datasets. Inline with previous studies, we binarized calcium imaging datasets by setting to 1 deflections in calcium concentration that exceeded three standard deviations above the mean [37]. The standard method for avalanche detection fails for brain-wide cellular resolution data because the large number of cells produce a single never-ending avalanche. Inline with recent work, we used a method to detect spatially contiguous avalanches from binarized zebrafish calcium imaging timeseries [37]. More specifically, we detected clusters of simultaneously active and spatially contiguous neu- 
rons. We used the MATLAB function bwconncomp to find these clusters. We then formed neuronal avalanches by detecting clusters that shared cells at adjacent timepoints. In this way, we followed the spatiotemporal structure of each avalanche as it started, propagated and ended.

Power law fitting to avalanche distributions. We assessed the presence of power-law scaling in avalanche distributions, by adopting methods described in [14] and previously implemented in [90]. Specifically, we denoted the probability density function of avalanche size as $p(s)$

$$
p(s)=\frac{s^{-\tau}}{\zeta\left(\tau, s_{\min }\right)-\zeta\left(\tau, s_{\max }+1\right)}
$$

with a cumulative distribution function

$$
P(s)=\frac{\zeta(\tau, s)-\zeta\left(\tau, s_{\max }+1\right)}{\zeta\left(\tau, s_{\min }\right)-\zeta\left(\tau, s_{\max }+1\right)}
$$

where $s$ is avalanche size, $\tau$ is the scaling exponent, $s_{\min }$ and $s_{\max }$ are the lower and upper cut-off sizes and $\zeta(\tau, s)=\sum_{i=0}^{\infty}(i+s)^{\tau}$ is the generalized Hurwitz zeta function. The functions incorporate an upper cut-off $s_{\max }$ because all empirical distributions are necessarily bounded by system size [17]. We defined the probability distributions for avalanche durations $l$ in exactly the same way. We then estimated the exponents of avalanche sizes and durations using the method of maximum likelihood, as previously described [14, 90].

Exponent scaling and shape collapses. We quantified power-law exponents between mean avalanche size for a given avalanche duration. We estimated the slope of this power law using linear regression in log-log coordinates [70]. Finally, we quantified avalanche shape collapses by averaging all avalanches of a given duration in a single recording. We rescaled collapsed avalanche shapes to have unit area and avalanche durations to have length 1 . 


\section{References}

1. Buzsáki, G. Large-scale recording of neuronal ensembles. Nature Neuroscience 7, 446$451(2004)$.

2. He, B. J. Scale-free brain activity: past, present, and future. Trends in Cognitive Sciences 18, 480-487 (2014).

3. Breakspear, M. Dynamic models of large-scale brain activity. Nature Neuroscience 20, 340-352 (2017).

4. Palva, S. \& Palva, J. M. Roles of brain criticality and multiscale oscillations in temporal predictions for sensorimotor processing. Trends in Neurosciences 41, 729-743 (2018).

5. Deco, G., Jirsa, V. K. \& McIntosh, A. R. Emerging concepts for the dynamical organization of resting-state activity in the brain. Nature Reviews Neuroscience 12, 43-56 (2011).

6. Cunningham, J. P. \& Byron, M. Y. Dimensionality reduction for large-scale neural recordings. Nature Neuroscience 17, 1500-1509 (2014).

7. Churchland, M. M. et al. Neural population dynamics during reaching. Nature 487, 51$56(2012)$

8. Kato, S. et al. Global brain dynamics embed the motor command sequence of Caenorhabditis elegans. Cell 163, 656-669 (2015).

9. Shine, J. M. et al. Human cognition involves the dynamic integration of neural activity and neuromodulatory systems. Nature Neuroscience 22, 289-296 (2019).

10. Saxena, S. \& Cunningham, J. P. Towards the neural population doctrine. Current Opinion in Neurobiology 55, 103-111 (2019).

11. Trautmann, E. M. et al. Accurate estimation of neural population dynamics without spike sorting. Neuron 103, 292-308 (2019).

12. Buzsáki, G. \& Mizuseki, K. The log-dynamic brain: how skewed distributions affect network operations. Nature Reviews Neuroscience 15, 264-278 (2014).

13. Roberts, J. A., Boonstra, T. W. \& Breakspear, M. The heavy tail of the human brain. Current Opinion in Neurobiology 31, 164-172 (2015). 
14. Clauset, A., Shalizi, C. R. \& Newman, M. E. Power-law distributions in empirical data. SIAM Review 51, 661-703 (2009).

15. He, B. J., Zempel, J. M., Snyder, A. Z. \& Raichle, M. E. The temporal structures and functional significance of scale-free brain activity. Neuron 66, 353-369 (2010).

16. Donoghue, T. et al. Parameterizing neural power spectra into periodic and aperiodic components. Nature Neuroscience 23, 1655-1665 (2020).

17. Beggs, J. M. \& Plenz, D. Neuronal avalanches in neocortical circuits. Journal of Neuroscience 23, 11167-11177 (2003).

18. Gireesh, E. D. \& Plenz, D. Neuronal avalanches organize as nested theta-and beta/gammaoscillations during development of cortical layer 2/3. Proceedings of the National Academy of Sciences 105, 7576-7581 (2008).

19. Hahn, G. et al. Neuronal avalanches in spontaneous activity in vivo. Journal of Neurophysiology 104, 3312-3322 (2010).

20. Petermann, T. et al. Spontaneous cortical activity in awake monkeys composed of neuronal avalanches. Proceedings of the National Academy of Sciences 106, 15921-15926 (2009).

21. Shriki, O. et al. Neuronal avalanches in the resting MEG of the human brain. Journal of Neuroscience 33, 7079-7090 (2013).

22. Linkenkaer-Hansen, K., Nikouline, V. V., Palva, J. M. \& Ilmoniemi, R. J. Long-range temporal correlations and scaling behavior in human brain oscillations. Journal of Neuroscience 21, 1370-1377 (2001).

23. Wohrer, A., Humphries, M. D. \& Machens, C. K. Population-wide distributions of neural activity during perceptual decision-making. Progress in Neurobiology 103, 156-193 (2013).

24. Magnasco, M. O., Piro, O. \& Cecchi, G. A. Self-tuned critical anti-Hebbian networks. Physical Review Letters 102, 258102 (2009). 
25. Bialek, W. Perspectives on theory at the interface of physics and biology. Reports on Progress in Physics 81, 012601 (2017).

26. Cocchi, L., Gollo, L. L., Zalesky, A. \& Breakspear, M. Criticality in the brain: A synthesis of neurobiology, models and cognition. Progress in Neurobiology 158, 132-152 (2017).

27. Chialvo, D. R. Emergent complex neural dynamics. Nature Physics 6, 744-750 (2010).

28. Beggs, J. M. \& Timme, N. Being critical of criticality in the brain. Frontiers in Physiology 3, 163 (2012).

29. Kinouchi, O. \& Copelli, M. Optimal dynamical range of excitable networks at criticality. Nature Physics 2, 348-351 (2006).

30. Shew, W. L., Yang, H., Petermann, T., Roy, R. \& Plenz, D. Neuronal avalanches imply maximum dynamic range in cortical networks at criticality. Journal of Neuroscience 29, 15595-15600 (2009).

31. Beggs, J. M. \& Plenz, D. Neuronal avalanches are diverse and precise activity patterns that are stable for many hours in cortical slice cultures. Journal of Neuroscience $\mathbf{2 4 ,}$ $5216-5229$ (2004).

32. Zimmern, V. Why brain criticality is clinically relevant: a scoping review. Frontiers in Neural Circuits 14, 54 (2020).

33. Voytek, B. \& Knight, R. T. Dynamic network communication as a unifying neural basis for cognition, development, aging, and disease. Biological Psychiatry 77, 1089-1097 $(2015)$

34. Saggar, M. et al. Towards a new approach to reveal dynamical organization of the brain using topological data analysis. Nature Communications 9, 1-14 (2018).

35. Voytek, B. et al. Age-related changes in 1/f neural electrophysiological noise. Journal of Neuroscience 35, 13257-13265 (2015). 
36. Ezaki, T., Dos Reis, E. F., Watanabe, T., Sakaki, M. \& Masuda, N. Closer to critical resting-state neural dynamics in individuals with higher fluid intelligence. Communications Biology 3, 1-9 (2020).

37. Ponce-Alvarez, A., Jouary, A., Privat, M., Deco, G. \& Sumbre, G. Whole-brain neuronal activity displays crackling noise dynamics. Neuron 100, 1446-1459 (2018).

38. Solovey, G. et al. Loss of consciousness is associated with stabilization of cortical activity. Journal of Neuroscience 35, 10866-10877 (2015).

39. Maturana, M. I. et al. Critical slowing down as a biomarker for seizure susceptibility. Nature Communications 11, 1-12 (2020).

40. Stumpf, M. P. \& Porter, M. A. Critical truths about power laws. Science 335, 665-666 (2012).

41. Destexhe, A. \& Touboul, J. D. Is there sufficient evidence for criticality in cortical systems? eNeuro 8 (2021).

42. Jonas, E. \& Kording, K. P. Could a neuroscientist understand a microprocessor? PLoS Computational Biology 13, e1005268 (2017).

43. Krakauer, J. W., Ghazanfar, A. A., Gomez-Marin, A., MacIver, M. A. \& Poeppel, D. Neuroscience needs behavior: correcting a reductionist bias. Neuron 93, 480-490 (2017).

44. Bedard, C., Kroeger, H. \& Destexhe, A. Does the 1/f frequency scaling of brain signals reflect self-organized critical states? Physical Review Letters 97, 118102 (2006).

45. Haimovici, A., Tagliazucchi, E., Balenzuela, P. \& Chialvo, D. R. Brain organization into resting state networks emerges at criticality on a model of the human connectome. Physical Review Letters 110, 178101 (2013).

46. Touboul, J. \& Destexhe, A. Power-law statistics and universal scaling in the absence of criticality. Physical Review E 95, 012413 (2017).

47. Chaudhuri, R., He, B. J. \& Wang, X.-J. Random recurrent networks near criticality capture the broadband power distribution of human ECoG dynamics. Cerebral Cortex 28, 3610-3622 (2018). 
48. Rubinov, M. Constraints and spandrels of interareal connectomes. Nature Communications 7, 1-11 (2016).

49. Elsayed, G. F. \& Cunningham, J. P. Structure in neural population recordings: an expected byproduct of simpler phenomena? Nature Neuroscience 20, 1310-1318 (2017).

50. Yanagawa, T., Chao, Z. C., Hasegawa, N. \& Fujii, N. Large-scale information flow in conscious and unconscious states: an ECoG study in monkeys. PLOS ONE 8, e80845 (2013).

51. Johnson, E. L. et al. Dynamic frontotemporal systems process space and time in working memory. PLOS Biology 16, e2004274 (2018).

52. Goodale, S. E. et al. Resting-state SEEG may help localize epileptogenic brain regions. Neurosurgery 86, 792-801 (2020).

53. Mu, Y. et al. Glia accumulate evidence that actions are futile and suppress unsuccessful behavior. Cell 178, 27-43 (2019).

54. Parvizi, J. \& Kastner, S. Promises and limitations of human intracranial electroencephalography. Nature Neuroscience 21, 474-483 (2018).

55. Ahrens, M. B., Orger, M. B., Robson, D. N., Li, J. M. \& Keller, P. J. Whole-brain functional imaging at cellular resolution using light-sheet microscopy. Nature Methods 10, 413-420 (2013).

56. Prichard, D. \& Theiler, J. Generating surrogate data for time series with several simultaneously measured variables. Physical Review Letters 73, 951 (1994).

57. Liegeois, R., Laumann, T. O., Snyder, A. Z., Zhou, J. \& Yeo, B. T. Interpreting temporal fluctuations in resting-state functional connectivity MRI. Neuroimage 163, 437-455 (2017).

58. Palva, S. \& Palva, J. M. Discovering oscillatory interaction networks with M/EEG: challenges and breakthroughs. Trends in Cognitive Sciences 16, 219-230 (2012). 
59. Nagasaka, Y., Shimoda, K. \& Fujii, N. Multidimensional recording (MDR) and data sharing: an ecological open research and educational platform for neuroscience. PLOS ONE 6, e22561 (2011).

60. Gao, R., Peterson, E. J. \& Voytek, B. Inferring synaptic excitation/inhibition balance from field potentials. Neuroimage 158, 70-78 (2017).

61. Colombo, M. A. et al. The spectral exponent of the resting EEG indexes the presence of consciousness during unresponsiveness induced by propofol, xenon, and ketamine. Neuroimage 189, 631-644 (2019).

62. Lendner, J. D. et al. An electrophysiological marker of arousal level in humans. eLife 9, e55092 (2020).

63. Alonso, L. M. et al. Single-trial classification of awareness state during anesthesia by measuring critical dynamics of global brain activity. Scientific Reports 9, 1-11 (2019).

64. Schnider, T. W. et al. The influence of age on propofol pharmacodynamics. Anesthesiology 90, 1502-1516 (1999).

65. Solovey, G., Miller, K. J., Ojemann, J., Magnasco, M. O. \& Cecchi, G. A. Self-regulated dynamical criticality in human ECoG. Frontiers in Integrative Neuroscience 6, 44 (2012).

66. Alonso, L. M. et al. Dynamical criticality during induction of anesthesia in human ECoG recordings. Frontiers in Neural Circuits 8, 20 (2014).

67. Peterson, E. J., Rosen, B. Q., Campbell, A. M., Belger, A. \& Voytek, B. 1/f neural noise is a better predictor of schizophrenia than neural oscillations. bioRxiv, 113449 (2017).

68. Peng, C.-K., Havlin, S., Stanley, H. E. \& Goldberger, A. L. Quantification of scaling exponents and crossover phenomena in nonstationary heartbeat time series. Chaos $\mathbf{5}$, 82-87 (1995).

69. Touboul, J. \& Destexhe, A. Can power-law scaling and neuronal avalanches arise from stochastic dynamics? PLOS ONE 5, e8982 (2010).

70. Friedman, N. et al. Universal critical dynamics in high resolution neuronal avalanche data. Physical Review Letters 108, 208102 (2012). 
71. Sethna, J. P., Dahmen, K. A. \& Myers, C. R. Crackling noise. Nature 410, 242-250 (2001).

72. Bargmann, C. I. Beyond the connectome: how neuromodulators shape neural circuits. Bioessays 34, 458-465 (2012).

73. Marder, E. Neuromodulation of neuronal circuits: back to the future. Neuron 76, 1-11 (2012).

74. Jones, B. E. From waking to sleeping: neuronal and chemical substrates. Trends in pharmacological sciences 26, 578-586 (2005).

75. Lee, S.-H. \& Dan, Y. Neuromodulation of brain states. Neuron 76, 209-222 (2012).

76. Avery, M. C. \& Krichmar, J. L. Neuromodulatory systems and their interactions: a review of models, theories, and experiments. Frontiers in neural circuits 11, 108 (2017).

77. Honey, C. J. et al. Slow cortical dynamics and the accumulation of information over long timescales. Neuron 76, 423-434 (2012).

78. Murray, J. D. et al. A hierarchy of intrinsic timescales across primate cortex. Nature Neuroscience 17, 1661-1663 (2014).

79. Gao, R., van den Brink, R. L., Pfeffer, T. \& Voytek, B. Neuronal timescales are functionally dynamic and shaped by cortical microarchitecture. eLife 9, e61277 (2020).

80. Raut, R. V. et al. Global waves synchronize the brains functional systems with fluctuating arousal. Science Advances 7, eabf2709 (2021).

81. Laub, A. J. Matrix analysis for scientists and engineers (Siam, 2005).

82. Smith, R. L. Efficient Monte Carlo procedures for generating points uniformly distributed over bounded regions. Operations Research 32, 1296-1308 (1984).

83. Johnson, E. Intracranial EEG recordings of medial temporal, lateral frontal, and orbitofrontal regions in 10 human adults performing a visuospatial working memory task. 2018. http://crcns.org/data-sets/fcx/fcx-2. 
84. Oostenveld, R., Fries, P., Maris, E. \& Schoffelen, J.-M. FieldTrip: open source software for advanced analysis of MEG, EEG, and invasive electrophysiological data. Computational Intelligence and Neuroscience 2011 (2011).

85. Neumaier, A. \& Schneider, T. Estimation of parameters and eigenmodes of multivariate autoregressive models. ACM Transactions on Mathematical Software (TOMS) 27, 27-57 (2001).

86. Wen, H. \& Liu, Z. Separating fractal and oscillatory components in the power spectrum of neurophysiological signal. Brain Topography 29, 13-26 (2016).

87. Waschke, L. et al. Modality-specific tracking of attention and sensory statistics in the human electrophysiological spectral exponent. eLife $\mathbf{1 0}$ (eds Chait, M., Shinn-Cunningham, B. G., Postle, B. R. \& Simon, J. Z.) e70068. Issn: 2050-084X. https : //doi . org/10. 7554/eLife. 70068 (Oct. 2021).

88. Palva, J. M. et al. Neuronal long-range temporal correlations and avalanche dynamics are correlated with behavioral scaling laws. Proceedings of the National Academy of Sciences 110, 3585-3590 (2013).

89. Varley, T. F., Sporns, O., Puce, A. \& Beggs, J. Differential effects of propofol and ketamine on critical brain dynamics. PLOS Computational Biology 16, e1008418 (2020).

90. Rubinov, M., Sporns, O., Thivierge, J.-P. \& Breakspear, M. Neurobiologically realistic determinants of self-organized criticality in networks of spiking neurons. PLoS Computational Biology 7, e1002038 (2011). 


\section{Supplementary Files}

This is a list of supplementary files associated with this preprint. Click to download.

- sisubmit.pdf 